\title{
Sinc-Nyström Method for Numerical Solution of One-Dimensional Cauchy Singular Integral Equation Given on a Smooth Arc in the Complex Plane*
}

\author{
By Bernard Bialecki**and Frank Stenger
}

\begin{abstract}
We develop a numerical method based on Sinc functions to obtain an approximate solution of a one-dimensional Cauchy singular integral equation (CSIE) over an arbitrary, smooth, open $\operatorname{arc} L$ of finite length in the complex plane. At the outset, we reduce the CSIE to a Fredholm integral equation of the second kind via a regularization procedure. We then obtain an approximate solution to the Fredholm integral equation by means of Nyström's method based on a Sinc quadrature rule. We approximate the matrix and right-hand side of the resulting linear system by an efficient method of computing the Cauchy principal value integrals. The error of an $N$-point approximation converges to zero at the rate $O\left(e^{-c N^{1 / 2}}\right)$, as $N \rightarrow \infty$, provided that the coefficients of the CSIE are analytic in a region $D$ containing the $\operatorname{arc} L$ and satisfy a Lipschitz condition in $D$.
\end{abstract}

1. Introduction. In this paper we consider an application of the Sinc function method to the approximate solution of a Cauchy singular integral equation taken over a smooth, open $\operatorname{arc} L$ of finite length in the complex plane. The equation to be solved on $L$ has the form

$$
a w+b S w+K_{1} w=f_{1}
$$

where for $t \in L$,

$$
\begin{gathered}
S w(t)=\frac{1}{\pi i} f_{L} \frac{w(\tau) d \tau}{\tau-t}, \\
K_{1} w(t)=\int_{L} k_{1}(t, \tau) w(\tau) d \tau .
\end{gathered}
$$

The integral appearing in (1.2) is a Cauchy principal value integral defined by

$$
f_{L} \frac{w(\tau) d \tau}{\tau-t}=\lim _{\varepsilon \rightarrow 0^{+}} \int_{L_{\varepsilon}} \frac{w(\tau) d \tau}{\tau-t}
$$

where $L_{\varepsilon}$ is the part of $L$ obtained by deleting from $L$ all those points which are within a distance $\varepsilon$ from $t$. The complex functions $a, b, f_{1}$ and $k_{1}$ in Eq. (1.1) are assumed to be given on $L$ and it is required to find $w$, or an approximation to $w$.

Received August 18, 1987.

1980 Mathematics Subject Classification (1985 Revision). Primary 65R20; Secondary 45E05.

Key words and phrases. Cauchy singular integral equation.

* Research supported by U. S. Army Research Contract \#DAAL03-87-K-0008.

** Present address. Department of Mathematics, University of Kentucky, Lexington, Kentucky 40506-0027. 
Following Muskhelishvili $[12, \S 106]$ we assume that $a, b, k_{1}$ and $f_{1}$ satisfy a Lipschitz condition on $L\left(k_{1}\right.$ satisfies a Lipschitz condition with respect to both variables) and that the condition

$$
r(t) \equiv a^{2}(t)-b^{2}(t) \neq 0
$$

is satisfied on the closure $\bar{L}$ of $L$. We look for solutions $w$ of Eq. (1.1) belonging to the class $H^{*}[12, \S 77]$ (see also Definition 3.1 of this paper). The class $H^{*}$ consists of all functions which satisfy Lipschitz conditions everywhere on $L$ except at the endpoints, where integrable singularities are allowed.

Let $\kappa$ and $Z(t)$ be respectively the index and the fundamental function of Eq. (1.1) corresponding to the class $h_{0}[12, \S 109]$ (see also Subsection 2.3 of this paper). Then it is well known $[12, \S 109]$ that Eq. (1.1) is equivalent (in the sense that solutions $w \in H^{*}$ are being sought) to the following equation

$$
w+\frac{a}{r} K_{1} w-\frac{b Z}{r} S\left(\frac{K_{1} w}{Z}\right)=f_{2},
$$

where

$$
f_{2}=\frac{a f_{1}}{r}-\frac{b Z}{r} S\left(\frac{f_{1}}{Z}\right)+\frac{b Z}{r} P_{\kappa-1},
$$

and where in (1.7) $P_{\kappa-1}$ is an arbitrary polynomial of degree at most $\kappa-1\left(P_{\kappa-1} \equiv 0\right.$ if $\kappa \leq 0)$, provided that when $\kappa<0$ the additional conditions

$$
\int_{L} \frac{t^{j-1}\left(K_{1} w-f_{1}\right)(t) d t}{Z(t)}=0, \quad j=1(1)(-\kappa)
$$

are satisfied. Interchanging the order of integration in the last term on the left-hand side of Eq. (1.6), we rewrite Eq. (1.6) in the form $[12, \S 111]$

$$
w-K_{2} w=f_{2},
$$

where

$$
K_{2} w(t)=\int_{L}\left[\frac{b Z}{r}(t) \frac{1}{\pi i} f_{L} \frac{k_{1}(u, \tau) / Z(u)}{u-t} d u-\frac{a}{r}(t) k_{1}(t, \tau)\right] w(\tau) d \tau
$$

As it was observed in [3], [4], [5], [6], [8], [9], rather than solving Eq. (1.9) for $w \in H^{*}$, which may be unbounded at either or both endpoints of $L$, it is practically more convenient to solve for the function

$$
g=\frac{r w}{Z}
$$

which turns out to be bounded and continuous on $L$. Making use of the substitution (1.11), Eq. (1.9) becomes

$$
(I-K) g=f
$$

where $I$ is the identity operator and $K$ is a Fredholm integral operator defined by

$$
K g(t)=\int_{L} k(t, \tau) g(\tau) d \tau
$$

The kernel $k(t, \tau)$ in (1.13) is defined by

$$
k(t, \tau)=\frac{Z}{r}(\tau)\left[\frac{b(t)}{\pi i} f_{L} \frac{k_{1}(u, \tau) / Z(u)}{u-t} d u-\frac{a}{Z}(t) k_{1}(t, \tau)\right],
$$


and the term $f$ on the right-hand side of (1.12) is given by

$$
f=\frac{a f_{1}}{Z}-b S\left(\frac{f_{1}}{Z}\right)+b P_{\kappa-1} \text {. }
$$

The Fredholm integral equation of the second kind, (1.12)-(1.15), will be the starting point for the numerical method of this paper. We shall solve Eq. (1.12)-(1.15) approximately by means of Nyström's method based on a Sinc quadrature rule.

The paper has been organized into seven sections and two appendices. First, in Section 2 we recall some necessary background material related to the theory of the singular integral equation (1.1), so that the paper could be read independently. However, throughout the paper we quite frequently refer the reader to Muskhelishvili [12] for details of proofs. In Section 3 we examine closely the integral equation (1.12)-(1.15). First we show that the function $g$ defined by (1.11) is indeed a solution of Eq. (1.12)-(1.15) which is continuous and bounded on $L$ for any solution $w \in H^{*}$ of Eq. (1.1). Next we establish some properties of the kernel $k$ and right-hand side $f$ given by the formulas (1.14) and (1.15), respectively. In particular, we establish analyticity of $k$ in some domain $D$ containing $L$ under the assumption that the coefficients of Eq. (1.1) are analytic in $D$. We also determine, for fixed $t$ on $L$, the rate at which the kernel $k(t, \tau)$ becomes infinite as the second variable $\tau$ approaches either of the endpoints of $L$. These properties will later play an essential role in developing approximate methods for solving Eq. (1.12)-(1.15). In Section 4, which can be viewed as an independent part of the paper, we present Nyström's method based on a Sinc quadrature rule for the numerical solution of the general Fredholm integral equation of the second kind given by $(1.12),(1.13)$. Under appropriate assumptions on the kernel $k(t, \tau)$ of the operator $K$ and right-hand side $f$, we show that the error of convergence has exponential decay with respect to the number of nodes used in the quadrature rule. It turns out that this method is well suited for finding an approximate solution of Eq. (1.12)-(1.15), provided that we could accurately evaluate the kernel $k$ and right-hand side $f$ (see (1.14) and (1.15)), which in general are not known explicitly. Therefore, in Section 5 we first develop some effective quadrature formulas for the approximate evaluation of Cauchy principal value integrals given by (1.2). Finally, in Section 6 we combine results of the previous sections to describe an algorithm for the approximate solution of Eq. (1.12)-(1.15). We use formulas from Section 5 to evaluate the coefficients of the matrix and the components of the right-hand side vector in the linear system resulting from Nyström's method, and we show that the error is still of exponential decay. The final section contains two numerical examples on which our algorithm was tested.

We would like to stress that it is, of course, this exponential (rather than algebraic) rate of convergence which makes this method and the general Sinc function technique in numerical analysis so attractive (see the review paper [13]). Other known methods of solving Eq. (1.1) are based on collocation or Galerkin schemes. The rates of convergence of the error in these methods are of the order $O\left(n^{-c}\right)$, where $n$ denotes the number of function evaluations or basis functions, and where $c$ depends on the number of derivatives of the coefficients in Eq. (1.1) that exist and are uniformly bounded on $L$ (see [3], [4], [5], [8], [9], [10], [11], [14]). In addition, all other methods seem to deal only with singular integral equations (1.1) taken 
over the interval $(-1,1)$, whereas the method of this paper can be applied to more general $\operatorname{arcs} L$ in the complex plane.

Finally, we want to point out that the first important attempt to provide a foundation for the application of Sinc methods to the approximate solution of Eq. (1.1) taken over the interval $(-1,1)$ was made in [6], where the use of a somewhat complicated projection method was suggested. However, in the course of experiments and trials the authors have found that the Nyström method applied to the numerical solution of Eq. (1.12)-(1.15) is simple to implement, and it allows for carrying out a complete convergence and error analysis.

2. Preliminaries. In this section we state some definitions and results from the theory of singular integral equations, making this paper self-contained (for more details we refer the reader to Muskhelishvili's book [12]). We recall such notions as special and nonspecial ends of an arc $L$, the index and the fundamental function of Eq. (1.1) corresponding to the class $h_{0}$.

2.1. An Arc $L$ and the Class of Functions $\operatorname{Lip}(L)$. Throughout the paper we shall be assuming that $L=L\left(c_{1}, c_{2}\right)$ is a smooth, open arc of finite length in the complex plane with ends $c_{1}, c_{2}$ and direction from $c_{1}$ to $c_{2}$ (see [12] for precise definition and properties of such arcs). Here we want to point out that according to this definition, the points $c_{1}, c_{2}$ do not belong to $L$. The closure of arc $L=L\left(c_{1}, c_{2}\right)$ will be denoted by $\bar{L}$, i.e., $\bar{L}=L \cup\left\{c_{1}, c_{2}\right\}$.

Let us recall that a complex function $w(t)$ defined on $L$ satisfies a Lipschitz condition on $L$ with exponent $\mu(0<\mu \leq 1)$ if for all points $t_{1}, t_{2} \in L$ we have

$$
\left|w\left(t_{1}\right)-w\left(t_{2}\right)\right| \leq C\left|t_{1}-t_{2}\right|^{\mu}
$$

where $C$ is a positive constant independent of $t_{1}$ and $t_{2}$. We write $w \in \operatorname{Lip}(L)$ or $w \in \operatorname{Lip}_{\mu}(L)$ if we wish to emphasize the exponent $\mu$, and we shall say that $w$ is a function of the class $\operatorname{Lip}(L)$ or $\operatorname{Lip}_{\mu}(L)$, respectively. Let us note that if $w \in \operatorname{Lip}(L)$ then $w$ can be uniquely defined at the ends $c_{1}$ and $c_{2}$, so that $w \in \operatorname{Lip}(\bar{L})$. We shall always assume this to be the case when talking about $w \in \operatorname{Lip}(L)$.

2.2. Special and Nonspecial Ends of L. Let the coefficients $a$ and $b$ of Eq. (1.1) belong to $\operatorname{Lip}(L)$ and let the following condition be satisfied:

$$
r(t) \equiv a^{2}(t)-b^{2}(t) \neq 0, \quad t \in \bar{L} .
$$

For $t \in \bar{L}$ let us define the function $G$ by

$$
G(t)=\frac{a(t)-b(t)}{a(t)+b(t)},
$$

and let $\log G(t)$ be any definite one-valued branch of the logarithm which varies continuously on $\bar{L}$. Let us assume that $\alpha_{j}, \beta_{j}, j=1,2$, are real numbers such that

$$
\alpha_{j}+i \beta_{j}=\mp \frac{\log G\left(c_{j}\right)}{2 \pi i}
$$

where the minus sign is taken for $j=1$ and plus sign for $j=2$. Let integers $\lambda_{j}$ be selected so that

$$
-1<\alpha_{j}+\lambda_{j} \leq 0, \quad j=1,2 .
$$

If $-1<\alpha_{j}+\lambda_{j}<0$, then the corresponding end $c_{j}$ is called nonspecial. If $\alpha_{j}+\lambda_{j}=0$ or, equivalently, if $\alpha_{j}$ is an integer, then $c_{j}$ is a special end. Let us 
observe that if $c_{j}$ is a special end, then $\beta_{j}=0$ if and only if $b\left(c_{j}\right)=0$. Also, if $\operatorname{Im} a\left(c_{j}\right)=\operatorname{Re} b\left(c_{j}\right)=0$, then $c_{j}$ is a special end if and only if $b\left(c_{j}\right)=0$.

2.3. The Index and Fundamental Function of Class $h_{0}$. Let integers $\lambda_{j}$ be defined by (2.5). Then the number

$$
\kappa=-\sum_{j=1}^{2} \lambda_{j}
$$

is called the index of Eq. (1.1) corresponding to the class $h_{0}$. Let us recall (see [12, $\S 109]$ for details) that all possible solutions $w \in H^{*}$ of Eq. (1.1) can be divided into classes, depending on whether or not $w$ is to remain bounded in neighborhoods of one or more of the nonspecial ends of $L$. The class $h_{0}$ is the class for which no boundedness condition is imposed in any neighborhood of a nonspecial end of $L$. Thus this class actually consists of all solutions $w$ of Eq. (1.1) which belong to the space $H^{*}$.

Let

$$
\begin{gathered}
\Pi(z)=\prod_{j=1}^{2}\left(z-c_{j}\right)^{\lambda_{j}}, \\
\Gamma(z)=\frac{1}{2 \pi i} \int_{L} \frac{\log G(\tau) d \tau}{\tau-z}, \quad z \notin \bar{L},
\end{gathered}
$$

and let us set

$$
X(z)=\Pi(z) e^{\Gamma(z)}, \quad z \notin \bar{L} .
$$

Then for $t \in L$ the fundamental function $Z(t)$ of Eq. (1.1) corresponding to the class $h_{0}$ is defined by

$$
Z(t)=[a(t)+b(t)] X^{+}(t)=[a(t)-b(t)] X^{-}(t),
$$

where $X^{+}(t)$ and $X^{-}(t)$ denote limits of $X(z)$ as $z$ approaches $t$ from the left and right of $L$, respectively. By the Plemelj formulas $[12, \S 17]$ it is seen from $(2.9)$ and (2.10) that

$$
Z(t)=[a(t)+b(t)] e^{\frac{1}{2} \log G(t)} \Pi(t) e^{\Gamma(t)}=[a(t)-b(t)] e^{-\frac{1}{2} \log G(t)} \Pi(t) e^{\Gamma(t)},
$$

where for $t \in L$,

$$
\Gamma(t)=\frac{1}{2 \pi i} f_{L} \frac{\log G(\tau) d \tau}{\tau-t}
$$

It can also be shown $[12, \S 107]$ that if we set

$$
\gamma_{j}=\alpha_{j}+\lambda_{j}+i \beta_{j}, \quad j=1,2,
$$

where $\alpha_{j}, \beta_{j}, \lambda_{j}$ are defined by (2.4) and (2.5), then

$$
Z(t)=\omega(t) \prod_{j=1}^{2}\left(t-c_{j}\right)^{\gamma_{j}}
$$

where $\omega$ is a function of the class $\operatorname{Lip}(L), \omega(t) \neq 0$ on $\bar{L}$, and where $\left(t-c_{j}\right)^{\gamma_{j}}$ is any definite one-valued branch of this function which varies continuously on $L$. It 
follows from (2.14) that the function $Z^{-1} \in \operatorname{Lip}\left(L^{\prime}\right)$ for any closed part $L^{\prime}$ of $L$. It is clear that if $c_{j}$ is a nonspecial end or special end with $b\left(c_{j}\right)=0$, then $Z^{-1}$ also satisfies a Lipschitz condition on $L$ near $c_{j}$ (in the first case $Z^{-1}\left(c_{j}\right)=0$ ). If, however, $c_{j}$ is a special end and $b\left(c_{j}\right) \neq 0$, then $Z^{-1}$ no longer satisfies a Lipschitz condition on $L$ near $c_{j}$, although it is bounded there.

2.4. The Fundamental Function in the Case of Real a and Purely Imaginary $b$. Let us assume that

$$
\operatorname{Im} a(t)=\operatorname{Re} b(t)=0, \quad t \in \bar{L},
$$

and let us introduce a continuous function $\Theta$, defined on $L$ by

$$
\Theta(t)=\frac{1}{\pi} \arg [a(t)+b(t)]+M,
$$

where $M$ is an integer. We note that

$$
\arg [a(t)+b(t)]=\arctan \left(\frac{\operatorname{Im} b(t)}{a(t)}\right)+\pi m(t),
$$

where $-\pi / 2<\arctan x<\pi / 2$ for $x \in \mathbf{R}$ and where $m(t)$ takes on only integer values and may have discontinuities at zeros of $a$. It follows from (2.16) and (2.3) upon setting

$$
\log G(t)=-2 \pi i \Theta(t)
$$

that we obtain on $L$ a definite, one-valued and continuous logarithm of the function $G$. Simple calculations, (2.16) and (2.18) also show that

$$
e^{-\frac{1}{2} \log G(t)}=(-1)^{M} \frac{a(t)+b(t)}{|a(t)+b(t)|} .
$$

Therefore, from (2.11), (2.12), (2.7), (2.18), (2.19), (2.2) and (2.15) we obtain

$$
Z(t)=(-1)^{M} r^{1 / 2}(t) \prod_{j=1}^{2}\left(t-c_{j}\right)^{\lambda_{j}} e^{-f_{L} \Theta(\tau) d \tau /(\tau-t)} .
$$

By (2.4), (2.5) and (2.18) we see that integers $\lambda_{j}, j=1,2$, appearing in (2.20) can now be chosen so that

$$
-1<\lambda_{j} \pm \Theta\left(c_{j}\right) \leq 0,
$$

where $\Theta$ is defined by (2.16), the plus sign is taken for $j=1$ and the minus for $j=2$. Let us note that if we set $M=0$ and omit the term $r^{1 / 2}$ on the rightithand side of (2.20), then we obtain a function which is called the fundamental solution of Eq. (1.1) by Dow and Elliott [3]. As can be seen from (2.21), the introduction of the integer $M$ in (2.16) allows for a broader choice of $\lambda_{j}$, which is of some importance if the formula (2.20) is used for computational purposes.

3. Properties of the Integral Equation (1.12)-(1.15). In this section we show that the function $g$ given by (1.11), which is a solution of Eq. (1.12)-(1.15), is bounded and continuous on $L$. We also establish some important properties of the kernel $k$ and right-hand side $f$ given by the formulas (1.14) and (1.15), respectively. In particular, we show that $k$ and $f$ are analytic functions ( $k$ with respect to each variable) in some domain $D$ containing $L$ if the coefficients of the 
singular integral equation (1.1) are analytic in $D$. We also determine the rate at which, for fixed $t$ from the arc $L$, the kernel $k(t, \tau)$ becomes infinite as the second variable $\tau$ approaches either of the endpoints of $L$. These facts will be essential for the development of a numerical method in Section 4.

3.1. Boundedness and Continuity of $g$. As was stated in Section 1 , the solution $w$ of Eq. (1.1) is being sought in the class $H^{*}$.

Definition $3.1[12, \S 77]$. The function $w(t)$, given on an $\operatorname{arc} L=L\left(c_{1}, c_{2}\right)$ such that $w \in \operatorname{Lip}\left(L^{\prime}\right)$ on every closed part $L^{\prime}$ of $L$, and near an endpoint $c=c_{1}$ or $c_{2}$ of the form

$$
w(t)=\frac{w^{*}(t)}{(t-c)^{\alpha}}, \quad 0 \leq \alpha<1,
$$

where $w^{*} \in \operatorname{Lip}(L)$, is said to belong to the class $H^{*}$.

We already mentioned in Section 1 that instead of solving Eq. (1.1) for $w \in H^{*}$ we shall look for the function $g$ related to $w$ by (1.11).

THEOREM 3.2. Let $a, b, k_{1}, f_{1}$ belong to $\operatorname{Lip}(L)$ ( $k_{1}$ with respect to both variables) and let condition (1.5) be satisfied on $\bar{L}$. Let $\kappa$ and $Z(t)$ be respectively the index and fundamental function of Eq. (1.1) corresponding to the class $h_{0}$. Then for any solution $w \in H^{*}$ of Eq. (1.1) the function $g$ defined by (1.11) is continuous and bounded on L. Furthermore, the function $g$ is a solution of Eq. (1.12)-(1.15), where in (1.15) $P_{\kappa-1}$ is a polynomial of degree not greater than $\kappa-1\left(P_{\kappa-1} \equiv 0\right.$, if $\kappa \leq 0)$.

Proof. It was shown in Section 1 that $g$ satisfies Eq. (1.12)-(1.15). Also, $g$ is continuous on $L$ since $Z$ is continuous on $L$ (see Subsection 2.3). Thus it remains to prove that $g$ is bounded on $L$. Rewriting Eq. (1.1), we have

$$
a w+b S w=f_{0},
$$

where $f_{0}=f_{1}-K_{1} w$. It is easy to show that $f_{0} \in \operatorname{Lip}(L)$. Therefore, from (1.6), (1.7) and (1.11) we obtain

$$
g=\frac{a f_{0}}{Z}-b S\left(\frac{f_{0}}{Z}\right)+b P_{\kappa-1} .
$$

It is obvious from $(2.14),(2.13)$ and $(2.5)$ that the first and last terms on the righthand side of (3.3) are bounded on $L$. Furthermore, $S\left(f_{0} / Z\right) \in \operatorname{Lip}\left(L^{\prime}\right)$ for every closed part $L^{\prime}$ of $L$. Hence, let us examine the behavior of $S\left(f_{0} / Z\right)$ on $L$ near the endpoints $c_{j}, j=1,2$. If $c_{j}$ is a nonspecial end, then $\left(f_{0} / Z\right)\left(c_{j}\right)=0$. It then follows from $(2.14),(2.13),(2.5)$ and the theorem in $\left[12, \S 19\right.$, p. 46] that $S\left(f_{0} / Z\right)$ satisfies a Lipschitz condition on $L$ near $c_{j}$ and hence is bounded there. On the other hand, if $c_{j}$ is a special end, then from the same equations of Section 2 and (29.7), (29.8) in $[12, \S 29]$ we observe that for $t \in L$ and $t$ in a neighborhood of $c_{j}$ we have

$$
S\left(\frac{f_{0}}{Z}\right)(t)=C_{1} \log \left(t-c_{j}\right)+s_{1}(t)
$$

if $b\left(c_{j}\right)=0$ and

$$
S\left(\frac{f_{0}}{Z}\right)(t)=\frac{C_{2}}{\left(t-c_{j}\right)^{i \beta_{j}}}+s_{2}(t)
$$


if $b\left(c_{j}\right) \neq 0$. In (3.4) and (3.5), $C_{1}, C_{2}$ are constants and $s_{1}(t)$ and $s_{2}(t)$ are functions that satisfy Lipschitz conditions on $L$ near $c_{j}$. If $b\left(c_{j}\right)=0$, then we see from (3.4), $5^{\circ}$ in $[12, \S 6]$ and $1^{\circ}$ in $[12, \S 7]$ that $b S\left(f_{0} / Z\right)$ satisfies a Lipschitz condition on $L$ near $c_{j}$ and hence is bounded there. If $b\left(c_{j}\right) \neq 0$, then (3.5) implies boundedness of $S\left(f_{0} / Z\right)$ on $L$ near $c_{j}$, and Theorem 3.2 follows.

The proof of Theorem 3.2 and results from Subsection 2.2 yield the following corollary.

COROLlaRY 3.3. If $\operatorname{Im} a\left(c_{j}\right)=\operatorname{Re} b\left(c_{j}\right)=0$ for $j=1,2$, in particular, if $a(t)$ is real and $b(t)$ purely imaginary on $L$, then $g \in \operatorname{Lip}(L)$.

3.2. Properties of the Kernel $k$ of (1.14) and the Right-Hand Side $f$ of (1.15). Let us assume that $D$ is a bounded, simply connected domain in the complex plane such that $D$ contains $L=L\left(c_{1}, c_{2}\right)$ and such that the points $c_{1}, c_{2}$ are boundary points of $D$. Let $D^{+}$and $D^{-}$denote the parts of $D$ lying respectively to the left and right-hand sides of $L$. Furthermore, let $\Omega$ be the union of two sets, $L \times D$ and $D \times L$. Throughout the paper, $H(D)$ will represent the set of all analytic functions in $D$ and $C(\bar{D})$ will denote the set of all continuous functions on the closure $\bar{D}$ of $D$. In what follows, whenever we say that a function $f$ defined on $L$ is in $H(D)$ and satisfies some additional properties on $D$ (such as, for example, $f$ is bounded on $D$ ), we will mean that $f$ has an analytic extension to $D$ with these properties. A similar convention will apply to a function of two variables defined on $L \times L$, if one of the variables is fixed and the function is considered as a function of only the other variable.

Assumption 3.4. Let $a, b \in \operatorname{Lip}(L) \cap H(D) \cap C(\bar{D})$ and let condition (1.5) be satisfied for all $t \in \bar{D}$.

LEMMA 3.5. Let Assumption 3.4 be satisfied, let $Z(t)$ be the fundamental function of Eq. (1.1) corresponding to the class $h_{0}$ and let $\alpha_{j}, \lambda_{j}, j=1,2$, be defined by (2.4) and (2.5). Then $Z, Z^{-1} \in H(D), Z^{-1}$ is bounded in $D$ and there exists a constant $C>0$ such that

$$
|Z(z)| \leq C\left(\left|z-c_{1}\right|\left|z-c_{2}\right|\right)^{\sigma-1}, \quad z \in D,
$$

where

$$
\sigma=1+\min _{j=1,2}\left(\alpha_{j}+\lambda_{j}\right)>0 .
$$

Proof. Let us recall that the fundamental function $Z(t)$ is defined for $t \in L$ by (2.10). If we extend $Z$ to $D$ by the formula

$$
Z(z)=X(z) \begin{cases}{[a(z)+b(z)],} & z \in D^{+}, \\ {[a(z)-b(z)],} & z \in D^{-}\end{cases}
$$

where $X(z)$ is given by $(2.9)$, then from the theorem on analytic continuation we see that $Z \in H(D)$. Furthermore, $Z^{-1} \in H(D)$ since $Z \neq 0$ on $D^{+} \cup D^{-}$by (3.8), (1.5), (2.9), and likewise $Z \neq 0$ on $L$ by (2.14). It is clear that in order to prove that $Z^{-1}$ is bounded in $D$ it will be sufficient to show that $Z^{-1}$ is bounded in $D$ near the points $c_{j}, j=1,2$. Proceeding similarly as in $[12, \S 79]$, it is easy to see that for $z \in D \backslash L$ and $z$ near $c_{j}$,

$$
X(z)=\left(z-c_{j}\right)^{\gamma_{j}} Y(z),
$$


where $\gamma_{j}$ is given by $(2.13),\left(t-c_{j}\right)^{\gamma_{j}}$ is any branch of this function defined in the complex plane cut along $L$, and $Y(z)$ is some function which is bounded and stays away from zero in the neighborhood of $c_{j}$. Boundedness of $Z^{-1}$ in $D$ near $c_{j}$ and the inequality (3.6) now follow from (3.8) and (3.9).

Assumption 3.6. Let $a, b$ be as in Assumption 3.4 and in addition let us assume that there exist positive constants $C$ and $\mu_{j}$ such that

$$
|b(z)| \leq C\left|z-c_{j}\right|^{\mu_{j}}, \quad z \in D,
$$

whenever $c_{j}$ is a special end of $L$, and $b\left(c_{j}\right)=0$.

We note that if both ends of $L$ are nonspecial, then Assumption 3.6 reduces to Assumption 3.4.

Assumption 3.7. In addition to satisfying a Lipschitz condition with respect to both variables on $L \times L$, let us assume that $k_{1}(\cdot, \tau) \in H(D)$ for all $\tau \in L$, $k_{1}(t, \cdot) \in H(D)$ for all $t \in L, k_{1}(t, \tau)$ is bounded on $\Omega$ and that there exist constants $C>0$ and $0<\mu \leq 1$ such that

$$
\left|k_{1}\left(t_{1}, \tau\right)-k_{1}\left(t_{2}, \tau\right)\right| \leq C\left|t_{1}-t_{2}\right|^{\mu}
$$

for all $t_{1}, t_{2} \in L$ and all $\tau \in D$.

LEMMA 3.8. Let Assumptions 3.6 and 3.7 be satisfied and for $(t, \tau) \in L \times L$ let $H(t, \tau)$ be defined by

$$
H(t, \tau)=b(t) f_{L} \frac{k_{1}(u, \tau) / Z(u)}{u-t} d u,
$$

where $Z$ is the fundamental function of Eq. (1.1) corresponding to the class $h_{0}$. Then $H(\cdot, \tau) \in H(D)$ for all $\tau \in L, H(t, \cdot) \in H(D)$ for all $t \in L$ and $H(t, \tau)$ is bounded in $\Omega$.

Proof. Two cases need to be considered.

I. The case when $\tau \in L$. For fixed $\tau \in L$ let the function $H(t, \tau)$, given for $t \in L$ by (3.12), be extended to $D$ with respect to $t$ as follows:

$$
H(t, \tau)=b(t)\left\{\begin{array}{cc}
-\pi i h(t, \tau)+\Phi(t, \tau), & t \in D^{+} \\
\pi i h(t, \tau)+\Phi(t, \tau), & t \in D^{-}
\end{array}\right.
$$

where

$$
h(t, \tau)=\frac{k_{1}(t, \tau)}{Z(t)}
$$

and where

$$
\Phi(t, \tau)=\int_{L} \frac{h(u, \tau) d u}{u-t}
$$

By Lemma 3.5 we know that $Z^{-1} \in H(D)$ and that $Z^{-1}$ is bounded in $D$. Hence $h(\cdot, \tau) \in H(D)$ and $h(t, \tau)$ is bounded for $t \in D$. The latter implies that $\Phi(\cdot, \tau)$ is analytic in $D^{+} \cup D^{-}$. Thus $H(\cdot, \tau)$ is also analytic in $D^{+} \cup D^{-}$. Next, applying Plemelj's formulas $[12, \S 17]$ to $\Phi(t, \tau)$ with respect to the variable $t$, we conclude via analytic continuation that $H(\cdot, \tau) \in H(D)$. To prove that $H(t, \tau)$ is bounded on $D \times L$, we note that it is sufficient to show boundedness of $H(t, \tau)$ for $\tau \in L$, $t \in D^{+} \cup D^{-}$and $t$ being near $c$, where $c$ is either of the endpoints $c_{1}, c_{2}$ of $L$. 
Boundedness for the remaining $t$ will then follow from the maximum principle. Also, since $b(t) h(t, \tau)$ is bounded on $D \times L$, we only need to consider the boundedness of $b(t) \Phi(t, \tau)$. The following argument will simplify our considerations. Let $L$ be divided into two parts, $L_{1}$ and $L_{2}$, such that $c \in \bar{L}_{1}$, and such that $L_{2}$ is at a finite distance from $c$. Let us split $\Phi(t, \tau)$ into the sum of two integrals, $\Phi_{1}(t, \tau)$ and $\Phi_{2}(t, \tau)$, which are taken respectively over $L_{1}$ and $L_{2}$. It is obvious that only $b(t) \Phi_{1}(t, \tau)$ has to be examined. Let us first assume that $c$ is either a nonspecial end, or else it is a special end with $b(c) \neq 0$. Then it follows from (2.14), (2.13) and $1^{\circ}, 2^{\circ}$ of Appendix A that $\Phi(t, \tau)$ is bounded for all $\tau \in L, t \in D^{+} \cup D^{-}$and $t$ being in the neighborhood of $c$. On the other hand, if $c$ is a special end with $b(c)=0$, then the boundedness of $b(t) \Phi(t, \tau)$ for the same $\tau$ and $t$ follows from $1^{\circ}$ in Appendix A and the inequality (3.10).

II. The case when $t \in L$. Let us define $\Psi(t, \tau)$ for fixed $t \in L$ and $\tau \in D$, by

$$
\Psi(t, \tau)=f_{L} \frac{h(u, \tau) d u}{u-t},
$$

where $h$ is given by (3.14). It is clear from (3.12) and (3.16) that in order to prove that $H(t, \cdot) \in H(D)$ and that $H(t, \tau)$ is bounded on $L \times D$, it will suffice to show that $\Psi(t, \cdot) \in H(D)$ and that $b(t) \Psi(t, \tau)$ is bounded on $L \times D$. First we note by $(12.4)$ of $[12, \S 12]$ that $(3.16)$ can be rewritten as

$$
\Psi(t, \tau)=\Psi_{1}(t, \tau)+h(t, \tau)\left[\pi i+\log \frac{c_{2}-t}{c_{1}-t}\right]
$$

where

$$
\Psi_{1}(t, \tau)=\int_{L} \frac{h(u, \tau)-h(t, \tau)}{u-t} d u
$$

It follows from (3.14) and (3.11) that for fixed $t \in L$ the integrand in (3.18) can be bounded from above, independently of $\tau \in D$, by a function that is integrable over $L$ with respect io $u$. Thus by Lebesgue's dominated convergence theorem, $\Psi_{1}(t, \cdot) \in$ $C(D)$. Now let $\Delta$ be a closed triangle such that $\Delta \subset D$. Since $h(t, \cdot) \in H(D)$, it follows by Fubini's theorem that $\int_{\partial \Delta} \Psi_{1}(t, \tau) d \tau=0$, and thus Morera's theorem implies that $\Psi_{1}(t, \cdot) \in H(D)$. Therefore, we conclude by (3.17) that $\Psi(t, \cdot) \in H(D)$. It remains to prove that $b(t) \Psi(t, \tau)$ is bounded on $L \times D$. It can be seen from (3.17) and (3.18) that it will be sufficient to show that $b(t) \Psi(t, \tau)$ is bounded for all $\tau \in D$, $t \in L$ and $t$ being in the neighborhood of $c$, where $c$ is either of the endpoints $c_{1}, c_{2}$ of $L$. But this follows from (3.16), (3.11) and $3^{\circ}, 4^{\circ}$ in Appendix A, by considering, as in case I above, the possibilities of $c$ being a nonspecial end, a special end with $b(c) \neq 0$ or a special end with $b(c)=0$.

THEOREM 3.9. Let Assumptions 3.6 and 3.7 be satisfied and let the kernel $k(t, \tau)$ of the operator $K$ of (1.13) be given by (1.14), for $(t, \tau) \in L \times L$. Then $k(\cdot, \tau) \in H(D)$ for all $\tau \in L$ and $k(t, \cdot) \in H(D)$ for all $t \in L$. Furthermore, there exists a constant $C>0$ such that

$$
|k(t, \tau)| \leq C\left(\left|\tau-c_{1}\right|\left|\tau-c_{2}\right|\right)^{\sigma-1}, \quad(t, \tau) \in \Omega,
$$

where $\sigma>0$ is given by (3.7).

Proof. The proof of Theorem 3.9 follows directly from (3.6) and Lemma 3.8.

Assumption 3.10. Let $f_{1} \in \operatorname{Lip}(L) \cap H(D)$ and let $f$ be bounded in $D$. 
THEOREM 3.11. Let Assumptions 3.6 and 3.10 be satisfied and let the righthand side $f$ of $E q$. (1.12) be given by $E q$. (1.15). Then $f \in H(D)$ and $f$ is bounded in $D$.

Proof. By Lemma 3.5, $Z^{-1} \in H(D)$ and $Z^{-1}$ is bounded in $D$. Hence it is seen from (1.15) that we only need to consider the term $b S\left(f_{1} / Z\right)$. But analyticity and boundedness in $D$ of this term follow from Lemma 3.8 upon setting $k_{1}(t, \tau)=f_{1}(t)$ for $(t, \tau) \in \Omega$.

4. Nyström's Method Based on a Sinc Quadrature Rule for the Numerical Solution of Fredholm Integral Equation (1.12)-(1.15). First, at the beginning of this section we shall recall briefly some definitions and results from the numerical theory of Sinc functions (see also [13]). Next, in Subsection 4.2 we present Nyström's method based on a Sinc quadrature rule to obtain an approximate solution of the integral equation $(1.12),(1.13)$. We want to emphasize that the results of Subsection 4.2 are independent of the rest of this paper and can be used for the numerical solution of a general Fredholm integral equation of the second kind given by $(1.12),(1.13)$. Finally, in Subsection 4.3 we show how the method of Subsection 4.2 can be applied to obtain an approximate solution of Eq. (1.12) (1.15), which was derived from the singular integral equation (1.1) via a regularization process.

4.1. The Sinc Quadrature Rule. Let $\mathbf{Z}, \mathbf{R}, \mathbf{C}$ denote, respectively, the set of all integers, the set of real numbers and the set of complex numbers, i.e., $\mathbf{Z}=\{n: n=$ $0, \pm 1, \ldots\}, \mathbf{R}=(-\infty, \infty), \mathbf{C}=\{z=x+i y: x \in \mathbf{R}, y \in \mathbf{R}\}$. Let $d>0$, and let us define $D_{d}$ by

$$
D_{d}=\{z \in \mathbf{C}:|\operatorname{Im} z|<d\} .
$$

Definition 4.1. Let $D$ be a simply connected, bounded domain in the complex plane $\mathbf{C}$ with boundary $\partial D$. Let $c_{1}, c_{2}\left(c_{1} \neq c_{2}\right)$ be boundary points of $D$ and let $\phi$ be a conformal map of $D$ onto $D_{d}$ (see (4.1)) such that $\phi\left(c_{1}\right)=-\infty, \phi\left(c_{2}\right)=\infty$. Let $\phi^{-1}$ denote the inverse map of $\phi$ and let the smooth open arc of finite length $L=L\left(c_{1}, c_{2}\right)$ be given by

$$
L=\left\{\phi^{-1}(x): x \in \mathbf{R}\right\} .
$$

In what follows, the set of all complex analytic functions in $D$ will be denoted by $H(D)$.

Definition 4.2. Let the domain $D$ be defined as in Definition 4.1. Then $B(D)$ will represent the family of all functions $F \in H(D)$ such that

$$
\begin{gathered}
\int_{\phi^{-1}\left(L_{x}\right)}|F(z) d z| \rightarrow 0 \quad \text { as } x \rightarrow \pm \infty, \\
N(F, D) \equiv \liminf _{y \rightarrow d^{-}} \int_{\phi^{-1}\left(L_{y}\right)}|F(z) d z|<\infty,
\end{gathered}
$$

where for $x \in \mathbf{R}$ and $0<y<d$,

$$
\begin{gathered}
L_{x}=\{z=x+i y:-d<y<d\}, \\
L_{y}=\{z=x \pm i y: x \in \mathbf{R}\} .
\end{gathered}
$$

The following result, obtained by Stenger [13, Theorem 4.4], will be of importance. 
THEOREM 4.3. Let $F \in B(D)$ and let there exist positive constants $C$ and $\alpha$ such that

$$
|F(t)| \leq C\left|\phi^{\prime}(t)\right| e^{-\alpha|\phi(t)|}, \quad t \in L .
$$

For a positive integer $N$ choose $h=[2 \pi d /(\alpha N)]^{1 / 2}$ and set $z_{n}=\phi^{-1}(n h), n=$ $-N(1) N$. Then

$$
\left|\int_{L} F(t) d t-h \sum_{n=-N}^{N} \frac{F\left(z_{n}\right)}{\phi^{\prime}\left(z_{n}\right)}\right| \leq e^{-(2 \pi d \alpha N)^{1 / 2}}\left[\frac{N(F, D)}{1-e^{-(2 \pi d \alpha N)^{1 / 2}}}+\frac{2 C}{\alpha}\right] .
$$

We shall refer to the summation formula in (4.8) as a Sinc quadrature rule.

4.2. Nyström's Method Based on a Sinc Quadrature Rule for the Numerical Solution of the General Fredholm Integral Equation of the Second Kind. Notations introduced in Subsection 4.1 will be used in this subsection. We shall assume in what follows that the domain $D$, the arc $L=L\left(c_{1}, c_{2}\right)$ and the map $\phi$ are given as in Definition 4.1. One more definition will also be useful.

Definition 4.4. Let $X$ be the Banach space of all continuous and bounded complex functions defined on $L$ normed with the sup norm, i.e., for $g \in X$,

$$
\|g\|_{X}=\sup _{t \in L}|g(t)|
$$

For $g \in X$ and $t \in L$, let

$$
K g(t)=\int_{L} k(t, \tau) g(\tau) d \tau
$$

be a bounded operator from $X$ to $X$, i.e., $\|K\|<\infty$, for which $(I-K)^{-1}$ exists on $X$ and hence is bounded. Then, for given $f \in X$, the equation

$$
(I-K) g=f
$$

has a unique solution $g \in X$ which will be found approximately by means of Nyström's method. For given positive integer $N, h>0, g \in X$ and $t \in L$, let $K_{N} g$ be defined by

$$
K_{N} g(t)=h \sum_{n=-N}^{N} \frac{k\left(t, z_{n}\right)}{\phi^{\prime}\left(z_{n}\right)} g\left(z_{n}\right)
$$

where

$$
z_{n}=\phi^{-1}(n h)
$$

Then the Nyström method for solving Eq. (4.11) is to find a solution $g_{N} \in X$ of the equation

$$
\left(I-K_{N}\right) g_{N}=f
$$

We shall show that under certain assumptions on $k(t, \tau)$, Eq. (4.14) has a unique solution for $N$ sufficiently large, and we shall bound the error $\left\|g-g_{N}\right\|_{X}$.

LEMMA 4.5. Let the following conditions be satisfied:

(a) $k(\cdot, \tau) \in H(D)$ for all $\tau \in L$;

(b) there exist positive constants $C$ and $\alpha$ such that for all $t \in D$ and all $\tau \in L$,

$$
|k(t, \tau)| \leq C\left|\phi^{\prime}(\tau)\right| e^{-\alpha|\phi(\tau)|}
$$


(c) let $K$ and $K_{N}$ be defined by (4.10) and (4.12), respectively.

Then for any $g \in X$ the functions $K g(t)$ and $K_{N} g(t)$ belong to $H(D)$; moreover,

$$
\begin{gathered}
\sup _{t \in D}|K g(t)| \leq \frac{2 C}{\alpha}\|g\|_{X}, \\
\sup _{t \in D}\left|K_{N} g(t)\right| \leq C\left(h+\frac{2}{\alpha}\right)\|g\|_{X} .
\end{gathered}
$$

Proof. For $g \in X$ and $t \in D$ we have from (4.10) and (4.15) that

$$
\begin{aligned}
|K g(t)| & \leq \int_{L}|k(t, \tau) g(\tau) d \tau| \leq C\|g\|_{X} \int_{L} e^{-\alpha|\phi(\tau)|}\left|\phi^{\prime}(\tau) d \tau\right| \\
& =C\|g\|_{X} \int_{-\infty}^{\infty} e^{-\alpha|x|} d x
\end{aligned}
$$

and thus (4.16) follows. It can also be seen from (4.18) that $k(t, \tau) g(\tau)$ is bounded from above, independently of $t$, by a function integrable over $L$. Hence by Lebesgue's dominated convergence theorem, $K g(t)$ is continuous in $D$. Next, applying Fubini's theorem, we also see that for any closed triangle $\Delta \subset D$ we have $\int_{\partial \Delta} K g(t) d t=0$. Thus, Morera's theorem implies that $K g(t) \in H(D)$. Similarly for (4.17): From (4.12), (4.13) and (4.15) we obtain

$$
\left|K_{N} g(t)\right| \leq h \sum_{n=-N}^{N}\left|\frac{k\left(t, z_{n}\right)}{\phi^{\prime}\left(z_{n}\right)} g\left(z_{n}\right)\right| \leq C h\|g\|_{X} \sum_{n=-N}^{N} e^{-\alpha|n h|} .
$$

Since for $\alpha, h>0$ we have $e^{\alpha h}-1>\alpha h$, (4.17) follows after evaluating and estimating the sum on the right-hand side of (4.19).

COROLlaRY 4.6. $K$ is a bounded operator from $X$ to $X$ with $\|K\| \leq 2 C / \alpha$.

COROLlaRY 4.7. $K_{N}$ is a compact operator from $X$ to $X$ with $\left\|K_{N}\right\| \leq$ $C(h+2 / \alpha)$.

LEMMA 4.8. Let the following conditions be satisfied:

(a) $k(t, \cdot) \in H(D)$ for all $t \in L$;

(b) the inequality (4.15) is satisfied for all $t \in L$ and for all $\tau \in D$;

(c) $h$ in (4.12) and (4.13) is selected by $h=[2 \pi d /(\alpha N)]^{1 / 2}$.

Then for any $g \in H(D)$ and $g$ bounded in $D$,

$$
\left\|\left(K-K_{N}\right) g\right\|_{X} \leq e^{-(2 \pi d \alpha N)^{1 / 2}}\left[\frac{2}{1-e^{-(2 \pi d \alpha N)^{1 / 2}}}+1\right] \frac{2 C}{\alpha} \sup _{z \in D}|g(z)| .
$$

Proof. Let $M=\sup _{z \in D}|g(z)|$. For fixed $t \in L$ let us consider the function $F(z)=k(t, z) g(z)$, where $z \in D$. Then it follows from assumption (b) that

$$
|F(z)| \leq M C\left|\phi^{\prime}(z)\right| e^{-\alpha|\phi(z)|}, \quad z \in D .
$$

We also observe that $F \in B(D)$, since (4.3) and (4.4) follow from (4.21). For example, we have

$$
N(F, D) \leq M C N\left(e^{-\alpha|\phi(z)|}\left|\phi^{\prime}(z)\right|, D\right)=2 M C \int_{-\infty}^{\infty} e^{-\alpha|x+i d|} d x .
$$


Thus, since

$$
\left(K-K_{N}\right) g(t)=\int_{L} F(\tau) d \tau-h \sum_{n=-N}^{N} \frac{F\left(z_{n}\right)}{\phi^{\prime}\left(z_{n}\right)},
$$

Lemma 4.8 follows from Theorem 4.3, (4.21), (4.22) and (4.23).

THEOREM 4.9. Let all assumptions of Lemmas 4.5 and 4.8 be satisfied and let $(I-K)^{-1}$ exist on $X$. Then there is an integer $N_{0}>0$ such that for all integers $N>N_{0},\left(I-K_{N}\right)^{-1}$ exists on $X$ and

$$
\left\|\left(I-K_{N}\right)^{-1}\right\| \leq \frac{1+\left\|(I-K)^{-1}\right\|\left\|K_{N}\right\|}{1-\left\|(I-K)^{-1}\right\|\left\|\left(K-K_{N}\right) K_{N}\right\|} .
$$

Let $f \in H(D)$ and let $f$ be bounded in D. Let $g \in X$ and $g_{N} \in X$ be solutions of Eqs. (4.11) and (4.14), respectively. Then $g \in H(D)$ and $g$ is bounded in $D$. Furthermore, there exists a constant $C>0$ independent of $N$ and $g$ such that for $N>N_{0}$,

$$
\left\|g-g_{N}\right\|_{X} \leq C e^{-(2 \pi d \alpha N)^{1 / 2}} \sup _{z \in D}|g(z)| .
$$

Proof. To prove the first part of Theorem 4.9, let us take $g \in X$. Then it follows from Lemma 4.5 that $K_{N} g \in H(D)$ and that the inequality (4.17) holds true. Now applying Lemma 4.8 to $K_{N} g$, we have from (4.20) and (4.17) that

$$
\begin{aligned}
& \left\|\left(K-K_{N}\right) K_{N} g\right\|_{X} \\
& \quad \leq e^{-(2 \pi d \alpha N)^{1 / 2}} \frac{2 C^{2}}{\alpha}\left[\frac{2}{1-e^{-(2 \pi d \alpha N)^{1 / 2}}}+1\right]\left(h+\frac{2}{\alpha}\right)\|g\|_{X} .
\end{aligned}
$$

But the inequality (4.26) implies that $\left\|\left(K-K_{N}\right) K_{N}\right\| \rightarrow 0$ as $N \rightarrow \infty$ and hence the first part of Theorem 4.9 follows from Theorem 1.10 in $[1, \S 1.7]$ and Corollaries 4.6 and 4.7. To prove the second part of Theorem 4.9, we first observe that the identity $g=K g+f$ and Lemma 4.5 imply that $g \in H(D)$ and that $g$ is bounded in $D$. To verify (4.25), we note from (4.11) and (4.14) that

$$
g-g_{N}=\left(I-K_{N}\right)^{-1}\left(K-K_{N}\right) g
$$

and hence

$$
\left\|g-g_{N}\right\|_{X} \leq\left\|\left(I-K_{N}\right)^{-1}\right\|\left\|\left(K-K_{N}\right) g\right\|_{X} .
$$

Now using (4.24), we see that the first factor on the right-hand side of (4.28) can be estimated by a constant as $N \rightarrow \infty$, since $\left\|\left(K-K_{N}\right) K_{N}\right\| \rightarrow 0$ and since, by Corollary 4.7, $\left\|K_{N}\right\|$ can be bounded independently of $N$. Thus, (4.25) follows from (4.28) and (4.20).

In order to find the solution $g_{N}$ of Eq. (4.14), assuming of course that $N>N_{0}$, we first solve the linear system

$$
(\mathbf{I}-\mathbf{K}) \mathbf{d}=\mathbf{f}
$$

for the vector $\mathbf{d}=\left(d_{-N}, \ldots, d_{N}\right)^{t}$, where in (4.29) I denotes the unit matrix of order $2 N+1$, and where the square matrix $\mathbf{K}=\left(k_{m n}\right)_{m, n=-N}^{N}$ of the same order and the vector $\mathbf{f}=\left(f_{-N}, \ldots, f_{N}\right)^{t}$ are defined by

$$
k_{m n}=h \frac{k\left(z_{m}, z_{n}\right)}{\phi^{\prime}\left(z_{n}\right)}
$$




$$
f_{m}=f\left(z_{m}\right) .
$$

It follows from Theorem 4.9 that the linear system (4.29) has a unique solution. For, if $g_{N}$ is a solution of Eq. (4.14), then $\mathbf{d}=\left(g_{N}\left(z_{-N}\right), \ldots, g_{N}\left(z_{N}\right)\right)^{t}$ satisfies (4.29) and conversely, to each solution $\mathbf{d}$ of (4.29) there corresponds a unique solution $g_{N} \in X$ of Eq. (4.14) which agrees with the components of $\mathbf{d}$ at the points $z_{n}$, $n=-N(1) N$ (see $[2, \S 3.0]$ ). Thus, having found a solution $\mathbf{d}$ of (4.29), we have

$$
g_{N}\left(z_{n}\right)=d_{n}, \quad n=-N(1) N
$$

and hence from (4.14) and (4.12),

$$
g_{N}(t)=f(t)+h \sum_{n=-N}^{N} \frac{k\left(t, z_{n}\right)}{\phi^{\prime}\left(z_{n}\right)} d_{n}, \quad t \in L .
$$

4.3. The Numerical Solution of Eq. (1.12)-(1.15). We now consider applicability of Nyström's method of Subsection 4.2 to obtain an approximate solution of Eq. (1.12)-(1.15). Let us assume that the arc $L=L\left(c_{1}, c_{2}\right)$ and the domain $D$ are defined as in Definition 4.1, and let Assumptions 3.6, 3.7 and 3.10 be satisfied. In addition, assume that for the map $\phi$ of Definition 4.1 and for any $\alpha>0$ there is a positive constant $C$ such that

$$
\left(\left|z-c_{1}\right|\left|z-c_{2}\right|\right)^{\alpha-1} \leq C\left|\phi^{\prime}(z)\right| e^{-\alpha|\phi(z)|}, \quad z \in D .
$$

If $(I-K)^{-1}$ exists on $X$, then it follows from Theorems 3.9, 3.11, 4.9 and inequality (4.34) that Nyström's method of Subsection 4.2 with $\alpha=\sigma$, where $\sigma$ is given by (3.7), can be applied to obtain an approximate solution of Eq. (1.12)-(1.15). The inequality (4.25) then gives a bound on the error between the exact solution $g$ and its approximation $g_{N}$ defined in $(4.33)$, where $\mathbf{d}=\left(d_{-N}, \ldots, d_{N}\right)^{t}$ is a solution of the linear system (4.29). It is clear from the definitions of the kernel $k$, and the right-hand side $f$ (see (1.14) and (1.15)) that in order to evaluate the coefficients $k_{m n}$ (see (4.30)) of the matrix $\mathbf{K}$ and components $f_{m}$ (see (4.31)) of the vector $\mathbf{f}$ of the linear system (4.29), it will be necessary to have a good numerical technique for approximating singular integrals of the form (1.2). We therefore consider this problem before describing completely an algorithm for obtaining an approximate solution of Eq. (1.12)-(1.15).

\section{Quadrature Formulas for Evaluating Cauchy Principal Value Inte-}

grals. In this section we derive formulas for the approximate evaluation of singular integrals $S F(t), t \in L$, defined by (1.2) in the case when the function $F$ is analytic in some domain containing $L$. First we present a general, infinite sum formula, which will later be used to obtain a finite sum formula for a certain class of functions $F$. The formulas presented here were first introduced for specific arcs $L$ in $[6]$.

In this section we shall use the notations and definitions of Subsection 4.1. In what follows we assume that the arc $L=L\left(c_{1}, c_{2}\right)$, the domain $D$, the map $\phi$ and the constant $d$ are the same as those of Definition 4.1. Also, by $H(D)$ and $B(D)$ we denote the classes of functions introduced in Subsection 4.1.

5.1. General Quadrature Formula. For $h>0$ and $n \in \mathbf{Z}$ we define the functions

$$
t_{n}(z)=\frac{h}{\pi i} \frac{\cos [\pi\{\phi(z)-n h\} / h]-1}{\phi^{\prime}\left(z_{n}\right)\left(z-z_{n}\right)}, \quad z \in D
$$


where

$$
z_{n}=\phi^{-1}(n h)
$$

For later purposes we note that

$$
t_{n}\left(z_{m}\right)=\left\{\begin{array}{l}
\frac{h}{\pi i} \frac{(-1)^{m-n}-1}{\phi^{\prime}\left(z_{n}\right)\left(z_{m}-z_{n}\right)}, \quad \text { if } n \neq m \\
0, \quad \text { if } n=m .
\end{array}\right.
$$

LEMMA 5.1. Let the functions $t_{n}(z)$ be defined by Eq. (5.1). Then there is a constant $C>0$ such that for all $n \in \mathbf{Z}$

$$
\sup _{t \in L}\left|t_{n}(t)\right| \leq C
$$

Proof. See Appendix B.

THEOREM 5.2. Assume that a function $F \in B(D)$ is such that $\int_{L}|F(z) d z|<$ $\infty$, and let either one of the following inequalities (5.5) or (5.6) be satisfied:

$$
\begin{gathered}
\sum_{n \in \mathbf{Z}}\left|\frac{F\left(z_{n}\right)}{\phi^{\prime}\left(z_{n}\right)}\right|<\infty ; \\
\sum_{n \in \mathbf{Z}}\left|F\left(z_{n}\right)\right|<\infty .
\end{gathered}
$$

Given $t \in L$, let $S F(t)$ be defined by (1.2), and let $\varepsilon(t)$ be defined by

$$
\varepsilon(t)=S F(t)-\sum_{n \in \mathbf{Z}} F\left(z_{n}\right) t_{n}(t)
$$

where $t_{n}(t)$ are given by (5.1). Then

$$
|\varepsilon(t)| \leq N(F, D, t) \frac{e^{-\pi d /(2 h)}}{2 \pi \sinh [\pi d /(2 h)]},
$$

where

$$
N(F, D, t)=\liminf _{y \rightarrow d^{-}} \int_{\phi^{-1}\left(L_{y}\right)}\left|\frac{F(z)}{z-t} d z\right|,
$$

and where $L_{y}$ in (5.9) is defined by (4.6).

Proof. See Appendix B.

We remark that under our assumptions on $F$ in Theorem 5.2, the quantity $N(F, D, t)$ as well as $S F(t)$ may become unbounded as $t$ approaches either of the endpoints of $L$. Hence, in this case the estimate (5.8) should be interpreted in the sense of a relative error.

5.2. Uniform Estimate for $N(F, D, t)$. If the domain $D$ satisfies some additional geometric properties and $F(z)$ goes to zero fast enough as $z$ approaches the endpoints of the arc $L$, then it is possible to obtain a bound for $N(F, D, t)$ which is independent of $t$.

Assumption 5.3. Let $c$ denote either of the endpoints $c_{1}, c_{2}$ of $L$ and let $L_{y}$ be given by (4.6). For a constant $Y, 0<Y<d$, let positive constants $\varsigma_{l}, l=1,2,3$, exist such that

$$
|z-t| \geq \varsigma_{1}|t-c|
$$


for all $z \in \phi^{-1}\left(L_{y}\right)$, where $Y<|y|<d$, and for all $t \in L \cap D\left(c, \varsigma_{2}\right)$, where $D\left(c, \varsigma_{2}\right)=\left\{z \in \mathbf{C}:|z-c| \leq \varsigma_{2}\right\}$, and such that

$$
|z-t| \geq \varsigma 3
$$

for all $z \in \phi^{-1}\left(L_{y}\right)$, where $Y<|y|<d$, and for all $t \in L \backslash \bigcup_{j=1}^{2} D\left(c_{j}, \varsigma_{2}\right)$. In addition, let us also assume that for any $\alpha>0$, there exists a constant $C>0$ such that

$$
\int_{\phi^{-1}\left(L_{y}\right)}|z-c|^{\alpha-1}|d z|<C
$$

for all $y$ satisfying $Y<|y|<d$.

LEMMA 5.4. Let Assumption 5.3 be satisfied and let $F(z)$ be a complex-valued function defined on $D$ for which there exist positive constants $C$ and $\alpha$ such that

$$
|F(z)| \leq C\left(\left|z-c_{1}\right|\left|z-c_{2}\right|\right)^{\alpha}, \quad z \in D .
$$

Let $N(F, D, t)$ be defined by (5.9). Then

$$
\sup _{t \in L} N(F, D, t)<\infty .
$$

Proof. It is easy to see that the uniform boundedness of $N(F, D, t)$ for $t \in$ $L \backslash \bigcup_{j=1}^{2} D\left(c_{j}, \zeta_{2}\right)$ follows from (5.11), (5.13) and (5.12). Therefore, assume that $t \in L \cap D\left(c_{1}, \varsigma_{2}\right)$ (the case when $t \in L \cap D\left(c_{2}, \varsigma_{2}\right)$ is analogous) and let $y$ be such that $Y<|y|<d$. Then using (5.13), we obtain

$$
\int_{\phi^{-1}\left(L_{y}\right)}\left|\frac{F(z)}{z-t} d z\right| \leq C \sup _{z \in D}\left|z-c_{2}\right|^{\alpha} \int_{\phi^{-1}\left(L_{y}\right)} \frac{\left|z-c_{1}\right|^{\alpha}}{|z-t|}|d z|,
$$

where $\sup _{z \in D}\left|z-c_{2}\right|^{\alpha}$ is finite because $D$ is bounded. Now, since

$$
\begin{aligned}
& \int_{\phi^{-1}\left(L_{y}\right)} \frac{\left|z-c_{1}\right|^{\alpha}}{|z-t|}|d z| \pm \int_{\phi^{-1}\left(L_{y}\right)} \frac{\left|z-c_{1}\right|^{\alpha}}{\left|z-c_{1}\right|}|d z| \\
& \quad \leq\left|t-c_{1}\right| \int_{\phi^{-1}\left(L_{y}\right)} \frac{\left|z-c_{1}\right|^{\alpha-1}}{|z-t|}|d z|+\int_{\phi^{-1}\left(L_{y}\right)}\left|z-c_{1}\right|^{\alpha-1}|d z|
\end{aligned}
$$

the inequality (5.14) follows from (5.15), (5.16), (5.10) and (5.12).

5.3. Finite Sum Quadrature Formula. The next step in deriving effective formulas for approximating the singular integrals $S F(t)$ is to replace the infinite sum in (5.7) with a finite one.

Assumption 5.5. Let the domain $D$ and the map $\phi$ be defined as in Definition 4.1. In addition, assume that there exists a constant $C>0$ such that

$$
\left|z-c_{1}\right|\left|z-c_{2}\right| \leq C e^{-|\phi(z)|}, \quad z \in D,
$$

and such that

$$
\int_{\phi^{-1}\left(L_{x}\right)}|d z|<C, \quad \text { as } x \rightarrow \pm \infty
$$

where $L_{x}$ in (5.18), for $x \in \mathbf{R}$, is given by (4.5). 
THEOREM 5.6. Let Assumptions 5.3 and 5.5 be satisfied. Let $F \in H(D)$, and let us also assume that there exist positive constants $C$ and $\alpha$ such that

$$
|F(z)| \leq C\left(\left|z-c_{1}\right|\left|z-c_{2}\right|\right)^{\alpha}, \quad z \in D .
$$

Let $S F(t)$ be defined for $t \in L$ by (1.2), and let $h$ be selected for a positive integer $N$ by $h=[2 \pi /(\alpha N)]^{1 / 2}$. Then there exists a constant $C_{1}>0$, which is independent of $N$, such that for all $t \in L$,

$$
\left|S F(t)-\sum_{n=-N}^{N} F\left(z_{n}\right) t_{n}(t)\right| \leq C_{1} N^{1 / 2} e^{-(\pi d \alpha N)^{1 / 2}},
$$

where $t_{n}(t)$ and $z_{n}$ in (5.20) are defined by (5.1) and (5.2), respectively.

Proof. It follows from the assumptions that all hypotheses of Theorem 5.2 are satisfied. In particular, we see from (5.19) and (5.17) that there is a constant $C_{2}$ such that

$$
\sum_{n \in \mathbf{Z}}\left|F\left(z_{n}\right)\right| \leq C_{2} \sum_{n \in \mathbf{Z}} e^{-\alpha|n| h}<\infty
$$

and hence the inequality (5.6) holds. Next, by Lemmas 5.4, 5.1 and (5.8), (5.19), (5.17) we have for $t \in L$ that

$$
\left|S F(t)-\sum_{n=-N}^{N} F\left(z_{n}\right) t_{n}(t)\right| \leq C_{3} e^{-\pi d / h}+C_{4} \sum_{n=N+1}^{\infty} e^{-\alpha n h},
$$

where $C_{3}, C_{4}$ are positive constants independent of $N$ and $t$. The estimate (5.20) now follows from (5.22) upon choosing $h$.

COROLLARY 5.7. Let all assumptions of Theorem 5.6 except the inequality (5.19) be satisfied. Let numbers $F_{j}, j=1,2$, and constants $0<\alpha \leq 1$ and $C>0$ be such that

$$
\left|F(z)-F_{j}\right| \leq C\left|z-c_{j}\right|^{\alpha}, \quad z \in D,
$$

for $j=1,2$. Then there exists a constant $C_{1}>0$ which is independent of $N$ and such that for all $t \in L$,

$$
\left|S F(t)-S(L F)(t)-\sum_{n=-N}^{N}\left[F\left(z_{n}\right)-L F\left(z_{n}\right)\right] t_{n}(t)\right| \leq C_{1} N^{1 / 2} e^{-(\pi d \alpha N)^{1 / 2}}
$$

where

$$
L F(z)=\frac{c_{2}-z}{c_{2}-c_{1}} F_{1}+\frac{z-c_{1}}{c_{2}-c_{1}} F_{2}, \quad z \in D .
$$

Proof. From (5.25) and (5.23) we have for $z \in D$,

$$
\begin{array}{r}
|(F-L F)(z)| \leq\left|\frac{c_{2}-z}{c_{2}-c_{1}}\left[F(z)-F_{1}\right]\right|+\left|\frac{z-c_{1}}{c_{2}-c_{1}}\left[F(z)-F_{2}\right]\right| \\
\leq \frac{C}{\left|c_{2}-c_{1}\right|}\left|c_{2}-z\right|^{\alpha}\left|z-c_{1}\right|^{\alpha}\left(\left|c_{2}-z\right|^{1-\alpha}+\left|z-c_{1}\right|^{1-\alpha}\right) .
\end{array}
$$

Since $D$ is a bounded region, the functions $\left|c_{2}-z\right|^{1-\alpha}$ and $\left|z-c_{1}\right|^{1-\alpha}$ are uniformly bounded on $D$. Hence, there exists a constant $C_{2}>0$ such that

$$
|(F-L F)(z)| \leq C_{2}\left(\left|z-c_{1}\right|\left|z-c_{2}\right|\right)^{\alpha}, \quad z \in D .
$$


Thus, (5.24) follows upon applying Theorem 5.6 to $(F-L F)(z)$.

We note that $S(L F)(t)$ can be evaluated explicitly for $t \in L$, and hence (5.24) provides a very accurate formula for the approximate evaluation of $S F(t)$ for functions $F$ satisfying the assumptions of Corollary 5.7.

6. Algorithms and Error Estimates for the Numerical Solution of Eq. (1.1). Finally, we are in a position to present an algorithm to approximate the solution of Eq. (1.12)-(1.15), and hence also of Eq. (1.1). We shall assume that the arc $L=L\left(c_{1}, c_{2}\right)$ and the domain $D$ are given as in Definition 4.1, and that the inequality (4.34) and Assumptions 5.3 and 5.5 are satisfied. The coefficients $a, b, k_{1}, f_{1}$ of Eq. (1.1) will be assumed to satisfy Assumptions 3.6, 3.7 and 3.10, so that all results from Subsection 3.2 concerning the fundamental function $Z$ of Eq. (1.1) corresponding to the class $h_{0}$, the kernel $k$ (see (1.14)) and the righthand side $f$ (see (1.15)) of Eq. (1.12)-(1.15) will hold true. Let us also assume that for $K$ defined by $(1.13)$ the operator $(I-K)$ has an inverse on the space $X$ (see Definition 4.4). In presenting the algorithm, we consider two cases. First we describe and analyze in detail the algorithm when both endpoints of the arc $L=L\left(c_{1}, c_{2}\right)$ are nonspecial (see Subsection 2.2). Next, we treat the case when one or both of the endpoints are special. In what follows, we assume that the fundamental function $Z$ is known explicitly. Later, in Subsection 6.4, we explain how one can compute $Z$ approximately in the case when an explicit formula for $Z$ is not available.

6.1. Nonspecial Ends. In this subsection we assume that both ends $c_{1}, c_{2}$ of the arc $L=L\left(c_{1}, c_{2}\right)$ are nonspecial. The following additional result about the fundamental function $Z$ will be needed.

LEMMA 6.1. Let the assumptions of Lemma 3.5 be satisfied and let $c_{j}, j=1$ or 2 , be a nonspecial end of $L$, i.e., $-1<\alpha_{j}+\lambda_{j}<0$. Then there exists a constant $C_{j}>0$ such that

$$
\left|Z^{-1}(z)\right| \leq C_{j}\left|z-c_{j}\right|^{-\alpha_{j}-\lambda_{j}}, \quad z \in D
$$

Proof. Following the proof of Lemma 3.5, we see that (6.1) follows from (3.8), (3.9) and (1.5).

To simplify the derivation of the algorithm, let us set

$$
\alpha=\min \left(\sigma,-\alpha_{1}-\lambda_{1},-\alpha_{2}-\lambda_{2}\right)>0,
$$

where $\sigma, \alpha_{j}, \lambda_{j}, j=1,2$, are given by (3.7), (2.4) and (2.5). Then (3.6) and (6.1) imply that there exists a constant $C>0$ such that the fundamental function $Z$ satisfies

$$
|Z(z)| \leq C\left(\left|z-c_{1}\right|\left|z-c_{2}\right|\right)^{\alpha-1}, \quad\left|Z^{-1}(z)\right| \leq C\left(\left|z-c_{1}\right|\left|z-c_{2}\right|\right)^{\alpha}, \quad z \in D
$$

To obtain an approximate solution of Eq. (1.12)-(1.15), we use Nyström's method described in Subsection 4.2, with

$$
h=\left(\frac{2 \pi d}{\alpha N}\right)^{1 / 2}, \quad z_{n}=\phi^{-1}(n h) .
$$


We approximate the singular integrals in the expressions for $k_{m n}$ (see $(4.30),(1.14)$ ) and $f_{m}$ (see $(4.31),(1.15)$ ) by means of the summation formula in (5.20) with

$$
h^{*}=\left(\frac{\pi d}{\alpha 2 N}\right)^{1 / 2}, \quad z_{n}^{*}=\phi^{-1}\left(n h^{*}\right)
$$

and $2 N$ in place of $h, z_{n}$ and $N$, respectively. It is obvious from (6.4) and (6.5) that $h^{*}=h / 2$ and $z_{n}=z_{2 n}^{*}$. Therefore, on using (5.3), we obtain

$$
\begin{gathered}
\tilde{k}_{m n}=\frac{h}{\phi^{\prime}\left(z_{n}\right)} \frac{Z}{r}\left(z_{n}\right)\left[\frac{b\left(z_{m}\right) h^{*}}{\pi i} \sum_{\substack{l=-2 N \\
l \neq 2 m}}^{2 N} \frac{k_{1}\left(z_{l}^{*}, z_{n}\right) / Z\left(z_{l}^{*}\right)}{\phi^{\prime}\left(z_{l}^{*}\right)} \frac{1-(-1)^{l-2 m}}{z_{l}^{*}-z_{m}}\right. \\
\tilde{f}_{m}=\frac{a f_{1}}{Z}\left(z_{m}\right)-\frac{b\left(z_{m}\right) h^{*}}{\pi i} \sum_{\substack{l=-2 N \\
l \neq 2 m}}^{2 N} \frac{f_{1}\left(z_{l}^{*}\right) / Z\left(z_{l}^{*}\right) k_{1}\left(z_{m}, z_{n}\right)}{\phi^{\prime}\left(z_{l}^{*}\right)} \frac{1-(-1)^{l-2 m}}{z_{l}^{*}-z_{m}} \\
+b\left(z_{m}\right) P_{\kappa-1}\left(z_{m}\right)
\end{gathered}
$$

in place of $k_{m n}$ and $f_{m}$. It follows from Theorem 5.6 and the inequality (6.3) that

$$
\begin{gathered}
\left|k_{m n}-\tilde{k}_{m n}\right| \leq h \frac{\left(\left|z_{n}-c_{1}\right|\left|z_{n}-c_{2}\right|\right)^{\alpha-1}}{\left|\phi^{\prime}\left(z_{n}\right)\right|} O\left(N^{1 / 2} e^{-(2 \pi d \alpha N)^{1 / 2}}\right), \\
\left|f_{m}-\tilde{f}_{m}\right| \leq O\left(N^{1 / 2} e^{-(2 \pi d \alpha N)^{1 / 2}}\right) .
\end{gathered}
$$

Of course, the linear system (4.29) is now replaced by

$$
(\mathbf{I}-\tilde{\mathbf{K}}) \tilde{\mathbf{d}}=\tilde{\mathbf{f}}
$$

where $\tilde{\mathbf{d}}=\left(\tilde{d}_{-N}, \ldots, \tilde{d}_{N}\right)^{t}$ and where $\tilde{\mathbf{K}}=\left(\tilde{k}_{m n}\right)_{m, n=-N}^{N}$ and $\tilde{\mathbf{f}}=\left(\tilde{f}_{-N}, \ldots, \tilde{f}_{N}\right)^{t}$ are given by (6.6) and (6.7).

We shall use maximum vector and matrix norms, i.e.,

$$
|\mathbf{d}|_{\infty}=\max _{-N \leq n \leq N}\left|d_{n}\right|, \quad|\mathbf{K}|_{\infty}=\max _{-N \leq m \leq N} \sum_{n=-N}^{N}\left|k_{m n}\right|,
$$

to estimate the error between $\mathbf{d}$ and $\tilde{\mathbf{d}}$. By well-known results of linear algebra on solving perturbed linear systems (see for example [15]) we find that

$$
|\mathbf{d}-\tilde{\mathbf{d}}|_{\infty} \leq \frac{\left|(\mathbf{I}-\mathbf{K})^{-1}\right|_{\infty}}{1-\left|(\mathbf{I}-\mathbf{K})^{-1}\right|_{\infty}|\mathbf{K}-\tilde{\mathbf{K}}|_{\infty}}\left(|\mathbf{f}-\tilde{\mathbf{f}}|_{\infty}+|\mathbf{K}-\tilde{\mathbf{K}}|_{\infty}|\mathbf{d}|_{\infty}\right) .
$$

But for Nyström's method we have $\left|(\mathbf{I}-\mathbf{K})^{-1}\right|_{\infty} \leq\left\|\left(I-K_{N}\right)^{-1}\right\|$ (see $[2, \S 3.1]$ ), where $K_{N}$ is defined by (4.12). Hence it follows from (4.24), Corollary 4.7 and the fact that $\left\|\left(K-K_{N}\right) K_{N}\right\| \rightarrow 0$ as $N \rightarrow \infty$, that there exists a constant $C>0$, which is independent of $N$, such that

$$
\left|(\mathbf{I}-\mathbf{K})^{-1}\right|_{\infty} \leq C
$$


Now using (6.8), (6.9) and (4.34), we obtain from (6.12)

$$
|\mathbf{d}-\tilde{\mathbf{d}}|_{\infty}=O\left(N^{1 / 2} e^{-(2 \pi d \alpha N)^{1 / 2}}\right) .
$$

Therefore, from (4.25), (4.32), (6.14) and the triangle inequality we have

$$
\left|g\left(z_{n}\right)-\tilde{d}_{n}\right|=O\left(N^{1 / 2} e^{-(2 \pi d \alpha N)^{1 / 2}}\right), \quad n=-N(1) N,
$$

which gives the discrete error estimate between the exact values of the solution $g$ of Eq. (1.12)-(1.15) at the points $z_{n}$ and the corresponding components of the solution $\tilde{\mathbf{d}}$ of the linear system (6.10).

To find an approximation to $g(t)$ for arbitrary $t \in L$, we can make use of Eq. (4.33). First, let us replace $d_{n}$ by $\tilde{d}_{n}$, where $\tilde{\mathbf{d}}=\left(\tilde{d}_{-N}, \ldots, \tilde{d}_{N}\right)^{t}$ is a solution of the linear system (6.10). Next, we approximate the singular integrals in $k\left(t, z_{n}\right)$ and $f(t)$ (see (1.14) and (1.15)) by means of the summation formula in (5.20), where $h^{*}, z_{n}^{*}$ defined by $(6.5)$ and $2 N$ replace $h, z_{n}$ and $N$, respectively. Denoting by $\tilde{k}$ and $f$ the approximations to $k$ and $f$ obtained in this way, we see that $g_{N}$ in (4.33) is replaced by $\tilde{g}_{N}$, where

$$
\tilde{g}_{N}(t)=\tilde{f}(t)+h \sum_{n=-N}^{N} \frac{\tilde{k}\left(t, z_{n}\right)}{\phi^{\prime}\left(z_{n}\right)} \tilde{d}_{n}, \quad t \in L .
$$

Carrying out a similar error analysis as above, we can then show that

$$
\left|g(t)-\tilde{g}_{N}(t)\right|=O\left(N^{1 / 2} e^{-(2 \pi d \alpha N)^{1 / 2}}\right), \quad t \in L .
$$

Thus, it is seen from (6.15) and (6.17) that both discrete and continuous errors are of exponential decay with respect to $N$.

It follows from (6.16), that in order to evaluate $\tilde{g}_{N}(t)$ for each new value of $t \in L$, complicated expressions for $\tilde{f}(t)$ and $\tilde{k}\left(t, z_{n}\right)$ have to be computed. One way to overcome this inconvenience is to construct an interpolant to $g$, which would also give an exponential error estimate for all $t \in L$, and whose cost of evaluation for arbitrary $t \in L$ would be relatively low. To develop this idea further, we shall need the following result which establishes some properties of the function $g$.

LEMMA 6.2. Let Assumptions 3.4 and 3.10 be satisfied and let $k_{1}(t, \tau)$ satisfy a Lipschitz condition with respect to both variables on $L \times L$. Let us also assume that $k_{1}(\cdot, \tau) \in H(D)$ for all $\tau \in L$ and that $k_{1}(t, \tau)$ is bounded on $D \times L$. Let $0<\mu<1$ be such that $a, b, f_{1} \in \operatorname{Lip}_{\mu}(L)$ and let there exist positive constants $C_{1}$ and $C_{2}$ such that

$$
\left|k_{1}\left(t_{1}, \tau\right)-k_{1}\left(t_{2}, \tau\right)\right| \leq C_{1}\left|t_{1}-t_{2}\right|^{\mu}, \quad t_{1}, t_{2}, \tau \in L
$$

and

$$
\left|b(z)-b\left(c_{j}\right)\right| \leq C_{2}\left|z-c_{j}\right|^{\mu}, \quad z \in D,
$$

for $j=1,2$. Let both ends $c_{1}, c_{2}$ of the arc $L=L\left(c_{1}, c_{2}\right)$ be nonspecial and let $\alpha_{j}$ and $\lambda_{j}, j=1,2$, be given by (2.4) and (2.5). Then for any solution $w \in H^{*}$ of $E q$. (1.1) the function $g$ defined by (1.11) is bounded and analytic in D. Furthermore, $g$ is continuous at the ends $c_{1}$ and $c_{2}$ and there exists a constant $C_{3}>0$ such that

$$
\left|g(z)-g\left(c_{j}\right)\right| \leq C_{3}\left|z-c_{j}\right|^{\gamma}, \quad z \in D
$$


for $j=1,2$, where

$$
\gamma=\min \left(\mu,-\alpha_{1}-\lambda_{1},-\alpha_{2}-\lambda_{2}\right)>0 .
$$

Proof. First we show that for the fundamental function $Z$ we have $Z^{-1} \in$ $\operatorname{Lip}_{\gamma}(L)$. It is easy to see from our assumptions that the product of the first two factors on the right-hand sides of Eq. (2.11) is a function in the class $\operatorname{Lip}_{\mu}(L)$, $\operatorname{since}$ $\log G \in \operatorname{Lip}_{\mu}(L)$. Also it follows from Eqs. (2.7), (2.12) and the theorem stated in $[12, \S 19$, p. 46$]$ that $Z_{1}$ defined by $Z_{1}(t)=\Pi(t) e^{\Gamma(t)}$ satisfies $Z_{1}^{-1} \in \operatorname{Lip}_{\mu}\left(L^{\prime}\right)$, where $L^{\prime}$ is any closed subset of $L$. Therefore, it only remains to consider $Z_{1}^{-1}(t)$ on $L$ near the points $c_{1}$ and $c_{2}$. Without loss of generality we shall analyze $Z_{1}^{-1}(t)$ only for $t \in L$ and $t$ in a neighborhood of $c_{1}$. By adding and subtracting $\log G\left(c_{1}\right)$ in the numerator of $(2.12)$, it follows from $(2.7),(2.4)$, the identity

$$
\int_{L} \frac{d \tau}{\tau-t}=\log \left(c_{2}-t\right)-\log \left(t-c_{1}\right)
$$

(see $(12.9 \mathrm{a})$ in $[12, \S 12])$ and the theorem in $[12, \S 19$, p. 46] that

$$
Z_{1}(t)=\left(t-c_{1}\right)^{\alpha_{1}+\lambda_{1}+i \beta_{1}}\left(t-c_{2}\right)^{\lambda_{2}}\left(c_{2}-t\right)^{-\alpha_{1}-i \beta_{1}} e^{h(t)},
$$

where $h(t)$ is a function which satisfies a Lipschitz condition on $L$ near $c_{1}$ with exponent $\mu$. Thus, using results of $[12, \S 7]$, we conclude from Eq. (6.22) that $Z_{1}^{-1}$ satisfies a Lipschitz condition on $L$ near $c_{1}$ with exponent $\gamma$. This concludes the proof that $Z^{-1} \in \operatorname{Lip}_{\gamma}(L)$.

In order to verify the properties of $g$ stated in Lemma 6.2, we first note from Eq. (3.3) that

$$
g=g_{1}-g_{2}+g_{3}
$$

where

$$
g_{1}=\frac{a f_{0}}{Z}, \quad g_{2}=b S\left(\frac{f_{0}}{Z}\right), \quad g_{3}=b P_{\kappa-1}
$$

and where

$$
f_{0}(t)=f_{1}(t)-\int_{L} k_{1}(t, \tau) w(\tau) d \tau .
$$

It is easy to see from Eq. (6.25) and our assumptions on $f_{1}$ and $k_{1}$ that $f_{0}$ is bounded and analytic in $D$ and that $f_{0} \in \operatorname{Lip}_{\mu}(L)$. Hence it follows from Eq. (6.24), Lemma 3.5 and (6.1) that the function $g_{1}$ satisfies all assertions of Lemma 6.2. Obviously, the same is true for the function $g_{3}$. Let us therefore consider $h_{1}=S\left(f_{0} / Z\right)$, which can be extended analytically into $D$ via the formulas

$$
h_{1}(t)= \begin{cases}-h_{2}(t)+H(t), & t \in D^{+}, \\ h_{2}(t)+H(t), & t \in D^{-},\end{cases}
$$

where $h_{2}=f_{0} / Z$ and where $H(t)=(\pi i)^{-1} \int_{L} h_{2}(\tau) /(\tau-t) d \tau$. We conclude from (6.1) and from what has been proven about $f_{0}$ and $Z^{-1}$ that $h_{2} \in \operatorname{Lip}_{\gamma}(L)$ and also, that $h_{2}\left(c_{1}\right)=h_{2}\left(c_{2}\right)=0$. By the theorem in $[12, \S 22$, p. 53], the function $H$ satisfies all assertions of Lemma 6.2 in the regions $D^{+}$and $D^{-}$. Since the same is true for $h_{2}$, and since $g_{2}=b h_{1}$, it thus follows that $g$ has all the properties we set out to establish. 
We are now ready to construct an interpolant to $g$. Let us assume that all assumptions of Lemma 6.2 are satisfied, in addition to all assumptions listed at the beginning of this section. Let us set $\hat{h}=[\pi d /(\gamma M)]^{1 / 2}$, where $\gamma$ is given by (6.21) and $M$ is a positive integer, and let us define points $\hat{z}_{n}=\phi^{-1}(n \hat{h})$, where $n=-M(1) M$. Let

$$
S(n, \hat{h})(x)=\frac{\sin [\pi(x-n \hat{h}) / \hat{h}]}{\pi(x-n \hat{h}) / \hat{h}}, \quad x \in \mathbf{R},
$$

and let $\operatorname{Lg}(z)$ be the linear part of $g$, which is equal to $g$ at the endpoints $c_{1}, c_{2}$ of the $\operatorname{arc} L=L\left(c_{1}, c_{2}\right)$, i.e.,

$$
L g(z)=\frac{c_{2}-z}{c_{2}-c_{1}} g\left(c_{1}\right)+\frac{z-c_{1}}{c_{2}-c_{1}} g\left(c_{2}\right), \quad z \in D .
$$

Then it follows from Lemma 6.2 and Theorem 4.3 of [13], applied to $g-L g$, that

$$
\left|g(t)-L g(t)-\sum_{n=-M}^{M}[g-L g]\left(\hat{z}_{n}\right) S(n, \hat{h}) \circ \phi(t)\right| \leq C M^{1 / 2} e^{-(\pi d \gamma M)^{1 / 2}}
$$

for all $t \in L$, where $C$ depends only on $g, d$ and $\gamma$. Based on the inequality (6.29), the interpolant to $g$ can now be constructed as follows. First, from (6.16) we compute the approximations $\tilde{g}_{n}=\tilde{g}_{N}\left(\hat{z}_{n}\right)$ to $g$ at $\hat{z}_{n}$ for $n=-M(1) M$, for which we have

$$
\left|g\left(\hat{z}_{n}\right)-\tilde{g}_{n}\right| \leq O\left(N^{1 / 2} e^{-(2 \pi d \alpha N)^{1 / 2}}\right), \quad n=-M(1) M,
$$

by $(6.17)$. Next we set

$$
\mathcal{L} g(t)=\frac{c_{2}-t}{c_{2}-c_{1}} \tilde{g}_{-M}+\frac{t-c_{1}}{c_{2}-c_{1}} \tilde{g}_{M}, \quad t \in L,
$$

which is a linear function in $t$ equal to $\tilde{g}_{-M}$ and $\tilde{g}_{M}$ at $c_{1}$ and $c_{2}$, respectively. The interpolant to $g$ is then defined by

$$
\hat{g}(t)=\mathcal{L} g(t)+\sum_{n=-M}^{M}\left[\tilde{g}_{n}-\mathcal{L} g\left(\hat{z}_{n}\right)\right] S(n, \hat{h}) \circ \phi(t), \quad t \in L .
$$

Using (6.29), the triangle inequality and (6.30), it is easy to estimate the difference $(g-\hat{g})(t)$ for $t \in L$ by first estimating the expression $L g(t)-\mathcal{L} g(t)$.

Let us recall at this point that evaluations of $\tilde{g}_{n}=\tilde{g}_{N}\left(\hat{z}_{n}\right), n=-M(1) M$, can be carried out by means of Eq. (6.16). Then the construction of the interpolant $\hat{g}$ in (6.32) is simplified significantly if $\gamma$ and $M$ in the expression for the step $\hat{h}$ are equal, respectively, to $\alpha$ and $2 N$, which were used in the formula (6.4). Since we can always choose $\alpha$ of Eq. (6.2) and $\gamma$ of Eq. (6.21) by the expression

$$
\alpha=\gamma=\min \left(\sigma,-\alpha_{1}-\lambda_{1},-\alpha_{2}-\lambda_{2}, \mu\right)>0,
$$

we shall assume that $\gamma=\alpha$ and $M=2 N$. Then, of course, $\hat{h}=h / 2$, and it is easy to see that $\tilde{k}\left(\hat{z}_{2 m}, z_{n}\right)$ and $\tilde{f}\left(\hat{z}_{2 m}\right), m=-N(1) N$, in (6.16) will be equal, respectively, to $\tilde{k}_{m n}$ and $\tilde{f}_{m}$, from (6.6) and (6.7), since $\hat{h}=h^{*}$ and $\hat{z}_{n}=z_{n}^{*}, n=-2 N(1) 2 N$. This and (6.10) show in turn that $\tilde{g}_{2 m}=\tilde{d}_{m}, m=-N(1) N$, where values of $\tilde{d}_{m}$ have already been computed by solving the linear system (6.10). Also, the evaluation of $\tilde{g}_{m}$ for $m$ odd and $-2 N \leq m \leq 2 N$ is easy, since the corresponding values of $\tilde{k}\left(\hat{z}_{m}, z_{n}\right)$ and $\tilde{f}\left(\hat{z}_{m}\right)$ in Eq. (6.16) will be computed by formulas similar to 
those in (6.6) and (6.7). More precisely, let us replace $z_{m}$ and $2 m$ in (6.6) and (6.7) by $\hat{z}_{m}=z_{m}^{*}$ and $m$, respectively. Then the right-hand sides of (6.6) and (6.7) will yield $\tilde{k}\left(\hat{z}_{m}, z_{n}\right)$ and $\tilde{f}\left(\hat{z}_{m}\right)$, respectively. As we can see from (6.29) with $M$ replaced by $2 N$, another advantage of taking $\alpha$ equal to $\gamma$ and proceeding as above is that the difference between $g$ and its interpolant $\hat{g}$ (see (6.32)) will be of the same order as the error between $g$ and its approximation $\tilde{g}_{N}$ (see (6.16), (6.17)), which will be obtained via Nyström's method.

Since we are assuming for the time being that the fundamental function $Z$ is known explicitly, the approximation to the solution $w$ of Eq. (1.1) can be obtained from $(1.11)$, once the approximation to $g$ is known. Let $\tilde{w}$ be the approximation to $w$ obtained by replacing $g$ in (1.11) by either $\tilde{g}_{N}$ of $(6.16)$ or $\hat{g}$ of (6.32). Since, in general, $Z(t)$ will be unbounded at the ends of $L=L\left(c_{1}, c_{2}\right)$, the absolute error $|(w-\tilde{w})(t)|$ may be large in the neighborhoods of $c_{1}$ and $c_{2}$. However, if $g\left(c_{j}\right) \neq 0$, $j=1,2$, then $w(t)$ will become infinite for $t$ approaching $c_{1}$ or $c_{2}$, at the same rate as $Z(t)$ does. Thus, in this case the relative error $|(w-\tilde{w})(t) / w(t)|$ will be of the same order as the absolute error in the approximation of $g$ by $\tilde{g}_{N}$ or by $\hat{g}$.

6.2. Special Ends. In this subsection we assume that one or both of the endpoints of the arc $L=L\left(c_{1}, c_{2}\right)$ are special. Let us first establish some additional properties of the fundamental function $Z$.

LEMMA 6.3. Let the assumptions of Lemma 3.5 be satisfied and let $c_{j}, j=1$ or 2 , be a special end with $b\left(c_{j}\right)=0$, i.e., $\alpha_{j}+\lambda_{j}=0$. Let us assume that $0<\mu_{j} \leq 1$ is such that $a, b \in \operatorname{Lip}_{\mu_{j}}(L)$, the inequality (3.10) of Assumption 3.6 is satisfied, and that there exists a constant $C_{1}>0$ such that

$$
\left|a(z)-a\left(c_{j}\right)\right| \leq C_{1}\left|z-c_{j}\right|^{\mu_{j}}, \quad z \in D .
$$

Then $Z^{-1}$ is continuous at $c_{j}$, and there exists a constant $C_{2}>0$ such that

$$
\left|Z^{-1}(z)-Z^{-1}\left(c_{j}\right)\right| \leq C_{2}\left|z-c_{j}\right|^{\mu_{j}}, \quad z \in D .
$$

Proof. Without loss of generality we assume that $j=1$, i.e., that $c_{1}$ is a special end and that $b\left(c_{1}\right)=0$. Then it follows from Eq. (2.4) that $\log G\left(c_{1}\right)=-\alpha_{1} 2 \pi i$, where $\alpha_{1}$ is an integer. By adding and subtracting $\log G\left(c_{1}\right)$ in the numerator of (2.8) (compare [12, §16, Eq. (16.5)]) we obtain

$$
\Gamma(z)=\Gamma_{0}(z)-\alpha_{1} \log \frac{z-c_{2}}{z-c_{1}}, \quad z \notin \bar{L},
$$

where

$$
\Gamma_{0}(z)=\frac{1}{2 \pi i} \int_{L} \frac{h(\tau) d \tau}{\tau-z}, \quad h(\tau)=\log G(\tau)-\log G\left(c_{1}\right) .
$$

Here, $\log \left[\left(z-c_{2}\right) /\left(z-c_{1}\right)\right]$ is understood to be a definite branch of the logarithm which is one-valued in the complex plane cut along $L$. The assumption $a, b \in$ $\operatorname{Lip}_{\mu_{1}}(L)$ implies that both $\log G$ and $h$ are in the class $\operatorname{Lip}_{\mu_{1}}(L)$. Since obviously $h\left(c_{1}\right)=0$, it follows by the theorem in $\left[12, \S 16\right.$, p. 38] that $\Gamma_{0}(z)$ is continuous at $c_{1}$ and that $\Gamma_{0}(z)$ tends to $\Gamma_{0}\left(c_{1}\right)$ as $z \rightarrow c_{1}$. It can also be shown, by making use of the theorem in $[12, \S 22$, p. 53], that there is a constant $C>0$ such that for $z \notin \bar{L}$ and $z$ near $c_{1}$

$$
\left|\Gamma_{0}(z)-\Gamma_{0}\left(c_{1}\right)\right| \leq C\left|z-c_{1}\right|^{\mu_{1}} .
$$


Now using (6.36) as well as (2.9), (2.7) and the relation $\alpha_{1}+\lambda_{1}=0$, we obtain

$$
X(z)=\left(z-c_{2}\right)^{\lambda_{2}-\alpha_{1}} e^{\Gamma_{0}(z)}, \quad z \notin \bar{L} .
$$

Finally, since $b\left(c_{1}\right)=0$, we find from (2.10) and (6.39) that

$$
Z\left(c_{1}\right)=a\left(c_{1}\right) X\left(c_{1}\right) .
$$

Thus, (6.35) now follows from (3.8), (6.40), (6.39), (6.38) (6.34) and (3.10).

Without loss of generality let us now suppose that $c_{1}$ is a special end, and that $c_{2}$ is a nonspecial end. We also assume that $b\left(c_{1}\right)=0$, since the case $b\left(c_{1}\right) \neq 0$ is seldom encountered in applications, and even if it occurs, it can be handled by a similar, although somewhat more complicated, analysis.

In order to describe the algorithm, let us assume, in addition to all assumptions mentioned at the beginning of this section, that the assumptions of Lemma 6.3 corresponding to $j=1$ are also satisfied. Further, let us assume that the coefficients $k_{1}$ and $f_{1}$ of Eq. (1.1) satisfy inequalities similar to (6.34), with $j=1$. More precisely, let there exist a constant $C>0$ such that

$$
\begin{aligned}
& \left|k_{1}(z, \tau)-k_{1}\left(c_{1}, \tau\right)\right| \leq C\left|z-c_{1}\right|^{\mu_{1}}, \quad z \in D, \tau \in L, \\
& \left|f_{1}(z)-f_{1}\left(c_{1}\right)\right| \leq C\left|z-c_{1}\right|^{\mu_{1}}, \quad z \in D .
\end{aligned}
$$

Finally, set

$$
\alpha=\min \left(\sigma, \mu_{1},-\alpha_{2}-\lambda_{2}\right),
$$

where $\sigma, \alpha_{2}, \lambda_{2}$ are given by Eqs. (3.7), (2.4), (2.5). As in Subsection 6.1, we first apply Nyström's method with $h=[2 \pi d /(\alpha N)]^{1 / 2}$ and $z_{n}=\phi^{-1}(n h)$ to obtain an approximate solution of the integral equation (1.12)-(1.15). We approximate the singular integrals in $k_{m n}$ (see $\left.(4.30),(1.14)\right)$ and $f_{m}$ (see $(4.31),(1.15)$ ) via the summation formula in (5.24), in which we take $h^{*}=[\pi d /(\alpha 2 N)]^{1 / 2}, z_{n}^{*}=\phi^{-1}\left(n h^{*}\right)$ and $2 N$ in place of $h, z_{n}$ and $N$, respectively. We then get a linear system of the form $(\mathbf{I}-\tilde{\mathbf{K}}) \tilde{\mathbf{d}}=\tilde{\mathbf{f}}$. It is obvious from (5.24) that all error estimates from Subsection 6.1 still hold true. In particular, (6.17) will be satisfied if $\tilde{f}$ and $\tilde{k}$ in (6.16) are in this case approximations to $f$ and $k$, respectively, obtained by means of the summation formula in (5.24). Furthermore, we can show that an interpolant similar to the one in Eq. (6.32) can be constructed for $g$. Once again, an approximation to the solution $w$ of Eq. (1.1) will then be obtained via the relation (1.11).

Let us remark at this point that all cases considered so far, i.e., the ends of $L=L\left(c_{1}, c_{2}\right)$ being nonspecial as in Subsection 6.1 or else special, but with the coefficient $b$ vanishing at $c_{j}$, cover completely all possible situations which may occur when the coefficients $a$ and $b$ of Eq. (1.1) are respectively real and purely imaginary on $L$.

6.3. Determination of the Polynomial $P_{\kappa-1}$. We have tacitly assumed up to now that the polynomial $P_{\kappa-1}$ of degree not greater than $\kappa-1$, appearing on the right-hand side of Eq. (1.15), was known explicitly. In general, however, instead of $P_{\kappa-1}$ we may only know values of the solution $w$ to Eq. (1.1) at some discrete points $t_{j}, j=0(1) \kappa$, where $t_{j} \neq t_{l}$ for $j \neq l$. Hence, by the relation (1.11) we will also know values $g\left(t_{j}\right), j=0(1) \kappa$, of the solution $g$ to Eq. (1.12)-(1.15). Without 
loss of generality let us consider the case of nonspecial ends from Subsection 6.1, and let us assume that

$$
P_{\kappa-1}(z)=\sum_{j=0}^{\kappa-1} \gamma_{j} z^{j}
$$

where the $\gamma_{j}$ are unknown coefficients. Let the vectors $\tilde{\mathbf{d}}^{(j)}=\left(\tilde{d}_{-N}^{(j)}, \ldots, \tilde{d}_{N}^{(j)}\right)^{t}$ for $j=-1(1) \kappa-1$ be solutions of the linear system

$$
(\mathbf{I}-\tilde{\mathbf{K}}) \tilde{\mathbf{d}}^{(j)}=\tilde{\mathbf{f}}^{(j)},
$$

in which the matrices $\mathbf{I}$ and $\tilde{\mathbf{K}}$ are the same as in the linear system (6.10), and where the components $\tilde{f}_{m}^{(j)}, m=-N(1) N$, of the vectors $\tilde{\mathbf{f}}^{(j)}=\left(\tilde{f}_{-N}^{(j)}, \ldots, \tilde{f}_{N}^{(j)}\right)^{t}$ are given by

$$
\tilde{f}_{m}^{(j)}=b\left(z_{m}\right) z_{m}^{j}
$$

for $j=0(1) \kappa-1$, whereas the components $\tilde{f}_{m}^{(-1)}$ of the vector $\tilde{\mathbf{f}}^{(-1)}$ are equal to the sum of the first two terms on the right-hand side in (6.7). It follows from (6.44) that the solution $\tilde{\mathbf{d}}$ of the linear system (6.10) can now be written in the form

$$
\tilde{\mathbf{d}}=\tilde{\mathbf{d}}^{(-1)}+\sum_{j=0}^{\kappa-1} \gamma_{j} \tilde{\mathbf{d}}^{(j)}
$$

Thus having first solved (6.44) for $\tilde{\mathbf{d}}^{(j)}, j=-1(1) \kappa-1$, we can determine the coefficients $\gamma_{j}$ in (6.43) or (6.46) by equating either $\tilde{g}_{N}(t)$ or $\hat{g}(t)$, given respectively by (6.16) and (6.32), to $g(t)$ at the points $t=t_{j}, j=0(1) \kappa$. In the case when all discrete points $t_{j}, j=0(1) \kappa$, coincide with some of the points $z_{n}, n=-N(1) N$, it follows from the identity $\tilde{g}_{N}\left(z_{m}\right)=\tilde{d}_{m}$ that the coefficients $\gamma_{j}$ can be found easily from (6.46) by equating appropriate components of the vector on the right-hand side of (6.46) with those of the corresponding values of the function $g$. Once the vector $\tilde{\mathbf{d}}$ is determined, then an approximation to $g(t)$, for $t \in L$, is obtained using either (6.16) or (6.32).

6.4. Numerical Evaluation of the Fundamental Function $Z$. If the fundamental function $Z$ is not known explicitly, then in order to carry out the algorithms described in Subsections 6.1 and 6.2 , we will first have to evaluate $Z$ at some discrete points. For example, for the case of nonspecial ends, the values $Z\left(z_{n}^{*}\right)$, $n=-2 N(1) 2 N$, will be required, as we can see from Eqs. (6.6) and (6.7). It is obvious from Eq. (2.11) that the problem of computing $Z(t)$ for $t \in L$ leads to the evaluation of the function $\Gamma(t)$ given by (2.12). Let us assume that in addition to Assumption 3.4 we also have $a, b \in \operatorname{Lip}_{\mu}(D)$, i.e., $a$ and $b$ satisfy (2.1) for all $t_{1}, t_{2} \in D$. Then $\log G \in \operatorname{Lip}_{\mu}(D) \cap H(D)$, where $G$ is defined by (2.3). Hence, the function $\Gamma$ defined in (2.12) can be computed at the required points using the summation formula in (5.24).

7. Numerical Examples. In this section we consider two numerical examples which illustrate some of the points discussed in the preceding sections.

Example 7.1 [3]. Consider the singular integral equation

$$
-\left(1-t^{2}\right)^{1 / 2} w(t)+\frac{t}{\pi} \int_{-1}^{1} \frac{w(\tau) d \tau}{\tau-t}+\frac{1}{\pi} \int_{-1}^{1} \frac{w(\tau) d \tau}{\tau+t+3}=-1+\left(t^{2}+6 t+8\right)^{-1 / 2}
$$


for $-1<t<1$. This equation has the particular solution $w(t)=\left(1-t^{2}\right)^{-1 / 2}$. Both points -1 and 1 are nonspecial ends, the index $\kappa$ is 2 , and the fundamental function $Z$ is $Z(t)=2\left(1-t^{2}\right)^{-1 / 2}$. Thus it follows from Eqs. (1.5) and (1.11) that the exact solution $g$ of the Fredholm integral equation (1.12)-(1.15) is $g(t)=1 / 2$. Equation (1.12)-(1.15) has been solved approximately by the method of Subsection 6.1 with $\alpha=1 / 2$ and $d=\pi / 2$.

We have performed two tests for various values of $N$. In the first of these we used the explicit formula for the fundamental function $Z(t)$ to carry out computations, while in the second, the fundamental function $Z(t)$ was computed approximately, as described in Subsection 6.4, according to the formula (2.20). In both cases we have obtained two significant figures of accuracy in the approximate solution to $g\left(z_{n}\right), n=-N(1) N$, when $N=4$, three when $N=8$ and four when $N=16$. These results confirm the theoretical error estimate given by (6.15).

Example 7.2. Let us consider the singular integral equation

$$
\begin{aligned}
& (1-t)^{1 / 2} w(t)+\frac{(1+t)^{1 / 2}}{\pi} f_{-1}^{1} \frac{w(\tau) d \tau}{\tau-t}+\int_{-1}^{1}(t+\tau) w(\tau) d \tau \\
& =1+\frac{1}{\pi}\left(\frac{1+t}{1-t}\right)^{1 / 2} \ln \frac{1+((1-t) / 2)^{1 / 2}}{1-((1-t) / 2)^{1 / 2}}+2 \sqrt{2}(t+1 / 3)
\end{aligned}
$$

for $-1<t<1$. This equation has the solution $w(t)=(1-t)^{-1 / 2}$. The points -1 and 1 are respectively special and nonspecial ends, the index $\kappa$ is 0 and the fundamental function $Z$ is $Z(t)=(1-t)^{-1 / 2}$. The exact solution $g$ of Eq. (1.12)$(1.15)$ is $g(t)=2$. The approximate solution of Eq. (1.12)-(1.15) has been obtained by the method of Subsection 6.2, in which we chose $\alpha=1 / 2$ and $d=\pi / 2$. As in the previous example, corresponding to each value of $N$ we have set up the linear system arising from Nyström's method in two ways: firstly by making use of the explicit formula for the fundamental function and secondly by computing the fundamental function approximately. When computing approximations to $g\left(z_{n}\right)$ for $n=-N(1) N$ we have in both cases obtained one significant figure of accuracy when $N=4$, two when $N=8$ and three when $N=16$.

Appendix A. The Behavior of Cauchy Integrals Near the Ends of the Line of Integration in the Case when the Density Function Depends on a Parameter. Let $L=L\left(c_{1}, c_{2}\right)$ be a smooth, open arc of finite length with ends $c_{1}, c_{2}$ in the complex plane and with direction from $c_{1}$ to $c_{2}$, and let $\Lambda$ be a set of points in the complex plane. Let us assume that $w^{*}(t, \tau)$ is a bounded function defined on $L \times \Lambda$ which satisfies, uniformly for $\tau \in \Lambda$, a Lipschitz condition with respect to $t \in L$, i.e., there exist constants $C>0$ and $0<\mu \leq 1$ such that

$$
\left|w^{*}\left(t_{1}, \tau\right)-w^{*}\left(t_{2}, \tau\right)\right| \leq C\left|t_{1}-t_{2}\right|^{\mu},
$$

for all $t_{1}, t_{2} \in L$ and for all $\tau \in \Lambda$. Let us define the function $w$ for all $(t, \tau) \in L \times \Lambda$ by

$$
w(t, \tau)=\frac{w^{*}(t, \tau)}{(t-c)^{\gamma}}, \quad \gamma=i \beta,
$$

where $c$ is either $c_{1}$ or $c_{2}$, where $\beta$ is a real number and where $(t-c)^{\gamma}$ is any definite branch of this function which varies continuously on $L$. 
Let us state some results on the behavior of

$$
\Phi(z, \tau)=\int_{L} \frac{w(u, \tau) d u}{u-z}
$$

where $\tau \in \Lambda$. Let $z$ be near $c$, but not on $L$. Then:

$1^{\circ}$. If $\gamma=0$ (i.e., $w(t, \tau)=w^{*}(t, \tau)$ ), then

$$
\Phi(z, \tau)= \pm w(c, \tau) \log \frac{1}{z-c}+\Phi_{0}(z, \tau)
$$

where the upper sign is taken for $c=c_{1}$ and the lower one for $c=c_{2}$. In (A.4), $\log (1 /(z-c))=-\log (z-c)$ is any branch of the logarithm which is one-valued near $c$ in the complex plane cut along $L$ and the function $\Phi_{0}(z, \tau)$ is bounded for all $z$ in the neighborhood of $c$ and all $\tau \in \Lambda$, i.e., there exist positive constants $M$, $\delta$ such that

$$
\left|\Phi_{0}(z, \tau)\right| \leq M \quad \text { for }|z-c| \leq \delta, z \notin L, \tau \in \Lambda
$$

$2^{\circ}$. If $\gamma \neq 0$, then

$$
\Phi(z, \tau)= \pm \frac{\pi e^{ \pm \gamma \pi i}}{\sin (\gamma \pi)} \frac{w^{*}(c, \tau)}{(z-c)^{\gamma}}+\Phi_{0}(z, \tau)
$$

where the signs are chosen as in $1^{\circ}$. In (A.6), $(z-c)^{\gamma}$ is any branch of this function, one-valued near $c$ in the complex plane cut along $L$, taking the value $(t-c)^{\gamma}$ on the left side of $L$, and the function $\Phi_{0}(z, \tau)$ has the same properties as in $1^{\circ}$.

In the case when the point $z=t$ is on $L$ and near $c$ (of course, in this case the integral in (A.3) is to be understood as Cauchy principal value integral), we have the following results:

$3^{\circ}$. If $\gamma=0$, then $\Phi$ has the form

$$
\Phi(t, \tau)= \pm w(c, \tau) \log \frac{1}{t-c}+\Phi_{1}(t, \tau)
$$

where the signs are again chosen as in $1^{\circ}$, where $\log (1 /(t-c))=-\log (t-c)$ is any branch of the logarithm that is one-valued and continuous on $L$ and where $\Phi_{1}(t, \tau)$ is a bounded function for all $t \in L$ in the neighborhood of $c$ and all $\tau \in \Lambda$, i.e., there exist positive constants $M, \delta$ such that

$$
\left|\Phi_{1}(t, \tau)\right| \leq M \quad \text { for }|t-c| \leq \delta, t \in L, \tau \in \Lambda
$$

$4^{\circ}$. If $\gamma \neq 0$, then

$$
\Phi(t, \tau)= \pm \pi \cot (\gamma \pi) \frac{w^{*}(c, \tau)}{(t-c)^{\gamma}}+\Phi_{1}(t, \tau)
$$

where the signs are selected as in $1^{\circ}$ and where $\Phi_{1}(t, \tau)$ has the same properties as in $3^{\circ}$.

The above results were established in $[12, \S 29]$ for the special case of $w^{*}(t, \tau)=$ $w^{*}(t)$, i.e., when $w^{*}$ is independent of $\tau$. In our case the proofs may be carried out in a similar way, and we therefore omit them. 


\section{Appendix B. Proofs of Lemma 5.1 and Theorem 5.2.}

Proof of Lemma 5.1. It follows from the properties of smooth arcs that there exist positive constants $C_{1}$ and $C_{2}$ such that for all $n \in \mathbf{Z}$ and $|n|>C_{1}$ we have

$$
\int_{z_{n}}^{t}|d \tau| \leq C_{2}\left|t-z_{n}\right| \quad \text { for all } t \in l_{n}
$$

and

$$
\left|t-z_{n}\right| \geq \min _{t=z_{n-1}, z_{n+1}}\left|t-z_{n}\right| \text { for all } t \in L \backslash l_{n},
$$

where $l_{n}$ is the part of $L$ between $z_{n-1}$ and $z_{n+1}$, and where the integration in (B.1) is taken over the part of $L$ from $z_{n}$ to $t$. It is obvious from the definition of $t_{n}(z)$ that in order to prove Lemma 5.1, it will be sufficient to verify (5.4) for all $n \in \mathbf{Z}$ such that $|n|>C_{1}$. Thus, let $|n|>C_{1}$, and let us consider two cases, that when $t \in l_{n}$ and the other when $t \in L \backslash l_{n}$. In the case when $t \in l_{n}$, we find from (5.1), on using (B.1), that

$$
\begin{aligned}
\left|t_{n}(t)\right| & =\frac{h}{\pi}\left|\frac{1-\cos [\pi\{\phi(t)-n h\} / h]}{\phi^{\prime}\left(z_{n}\right)\left(t-z_{n}\right)}\right| \\
& =\left|\int_{z_{n}}^{t} \frac{\phi^{\prime}(\tau) \sin [\pi\{\phi(\tau)-n h\} / h] d \tau}{\phi^{\prime}\left(z_{n}\right)\left(t-z_{n}\right)}\right| \leq C_{2} \max _{z \in l_{n}}\left|\frac{\phi^{\prime}(z)}{\phi^{\prime}\left(z_{n}\right)}\right| \leq C_{2} I_{n}
\end{aligned}
$$

where

$$
I_{n}=\max _{\xi_{1}, \xi_{2} \in[(n-1) h,(n+1) h]}\left|\frac{\left(\phi^{-1}\right)^{\prime}\left(\xi_{1}\right)}{\left(\phi^{-1}\right)^{\prime}\left(\xi_{2}\right)}\right| .
$$

Similarly, when $t \in L \backslash l_{n}$, then from the obvious inequality $|1-\cos [\pi\{\phi(t)-n h\} / h]|$ $\leq 2$ and (B.2) we have

$$
\begin{aligned}
\left|t_{n}(t)\right| & \leq \frac{2}{\pi} \max _{t=z_{n-1}, z_{n+1}}\left|\frac{h}{\phi^{\prime}\left(z_{n}\right)\left(t-z_{n}\right)}\right| \\
& =\frac{2}{\pi} \max _{\xi=(n-1) h,(n+1) h}\left|\frac{\left(\phi^{-1}\right)^{\prime}(n h)}{\left[\phi^{-1}(\xi)-\phi^{-1}(n h)\right] / h}\right| \leq \frac{2}{\pi} I_{n},
\end{aligned}
$$

where $I_{n}$ is defined by (B.4). Thus we see from (B.3) and (B.5) that the quantity $I_{n}$ given by (B.4) needs to be estimated in both cases.

Let us introduce a domain $B_{0} \subset D_{d}$ which encloses the interval $[-h, h]$ on the $x$-axis; for simplicity, we have chosen $B_{0}$ to be a rectangle (see Figure B.6).

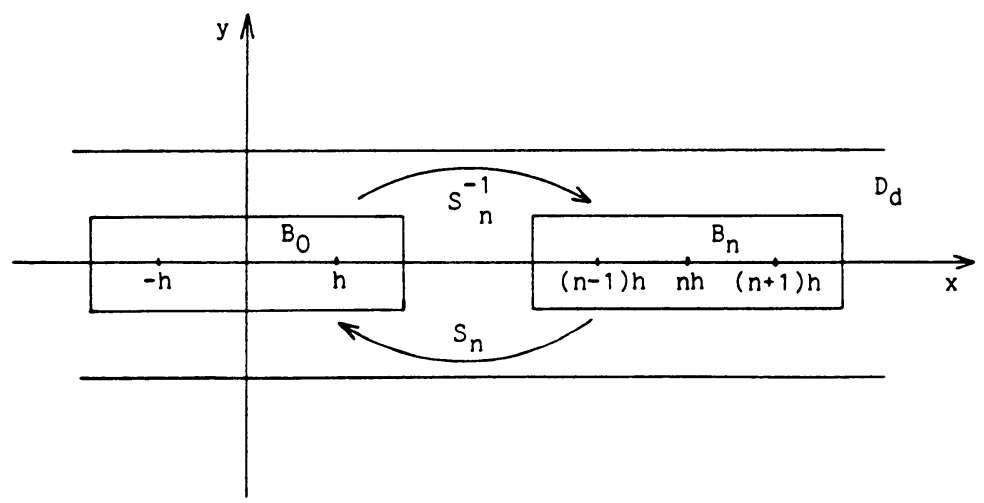

FiguRE B.6. The domain $B_{0}$. 
Let $s_{n}$ be the linear map such that $s_{n}((n-1) h)=-h$ and such that $s_{n}((n+1) h)=h$. Let $s_{n}^{-1}$ be the inverse map of $s_{n}$, and let $B_{n}=s_{n}^{-1}\left(B_{0}\right)$. Let us define a map $h_{n}$ on $B_{0}$ by $h_{n}(\xi)=\phi^{-1}\left(s_{n}^{-1}(\xi)\right)$. It is clear that $h_{n}$ is a conformal mapping of $B_{0}$ onto some subset of $D$. For $\xi \in B_{n}$ we have $\phi^{-1}(\xi)=h_{n}\left(s_{n}(\xi)\right)$ and hence

$$
\left(\phi^{-1}\right)^{\prime}(\xi)=h_{n}^{\prime}\left(s_{n}(\xi)\right) s_{n}^{\prime}(\xi) .
$$

Furthermore, since $s_{n}^{\prime}(\xi)=$ const $\neq 0$, Eq. (B.7) implies that

$$
I_{n}=\max _{\eta_{1}, \eta_{2} \in[-h, h]}\left|\frac{h_{n}^{\prime}\left(\eta_{1}\right)}{h_{n}^{\prime}\left(\eta_{2}\right)}\right| .
$$

Now using a result from the theory of univalent functions (see Theorem 4 from [7, $\S 4$, Chapter 2]), we conclude that a uniform bound, independent of $n$, exists for the right-hand side of (B.8). Hence Lemma 5.1 is proven.

Proof of Theorem 5.2. Let the inequality (5.5) be satisfied and let $t \in L$. Let $n$ be a positive integer such that $-n h \leq \phi(t) \leq n h$ and let $0<Y<d$. We introduce the following contours (see Figure B.10),

$$
\begin{aligned}
l_{n, Y}^{1} & =\phi^{-1}(\{z=x \pm i Y:-(n+2 / 3) h \leq x \leq(n+2 / 3) h\}), \\
l_{n, Y}^{2} & =\phi^{-1}(\{z= \pm(n+2 / 3) h+i y:-Y \leq y \leq Y\}) \\
l_{n, Y} & =l_{n, Y}^{1} \cup l_{n, Y}^{2} \\
L_{n} & =\phi^{-1}(\{z=x:-(n+1 / 3) h \leq x \leq(n+1 / 3) h\}) .
\end{aligned}
$$

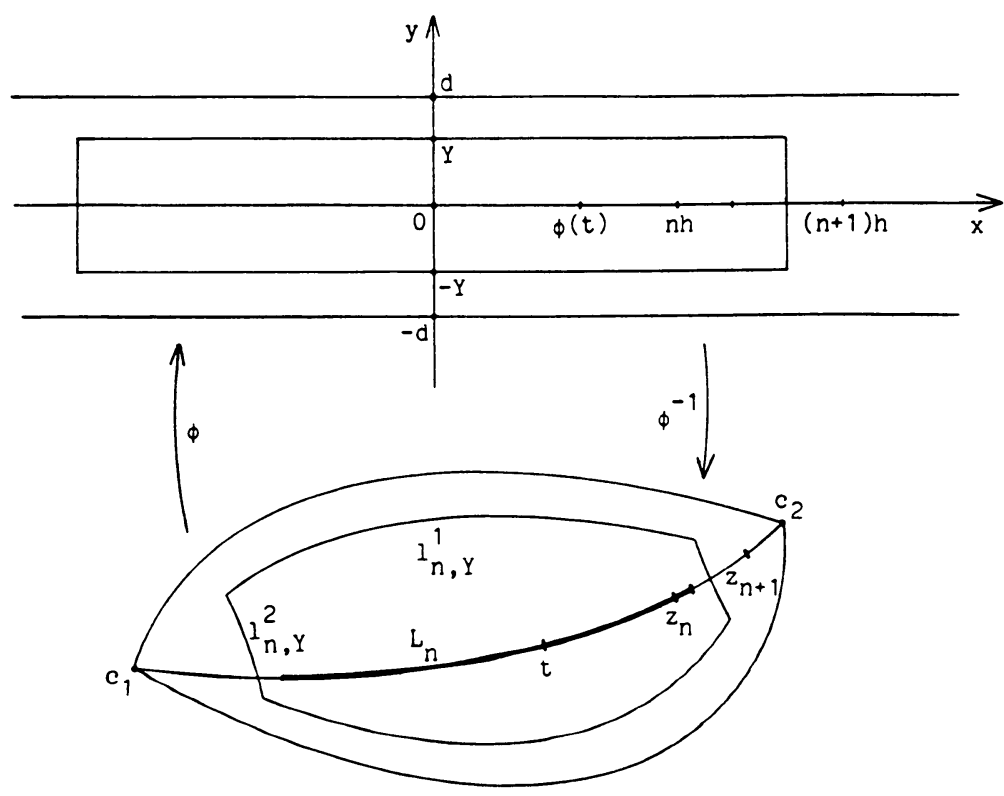

FIGURE B.10. The contours $l_{n, Y}^{1}, l_{n, Y}^{2}, L_{n}$.

In order to derive the formula for $\varepsilon(t)$ defined by (5.7), we assume that $t \neq z_{k}$ for all $k=-n(1) n$; the same formula for $t=z_{k}$ will follow by letting $t$ approach 
$z_{k}$. For any $\tau \in L_{n}$ and $\tau \neq t, \tau \neq z_{k}$ for all $k=-n(1) n$, we have from the residue theorem that

$$
\begin{gathered}
\frac{F(\tau)[\phi(\tau)-\phi(t)]}{i(\tau-t) \phi^{\prime}(\tau)}-\sum_{k=-n}^{n} \frac{F\left(z_{k}\right)}{\phi^{\prime}\left(z_{k}\right)} \frac{\phi(t)-k h}{t-z_{k}} \frac{h(-1)^{k}}{\pi i} \frac{\sin [\pi \phi(\tau) / h]}{\phi(\tau)-k h} \\
=-\frac{\sin [\pi \phi(\tau) / h]}{2 \pi} \int_{l_{n, Y}} \frac{F(z)[\phi(z)-\phi(t)] d z}{[\phi(z)-\phi(\tau)](z-t) \sin [\pi \phi(z) / h]} .
\end{gathered}
$$

Given $\varepsilon>0$, let $L_{n}^{\varepsilon}$ denote the image under the map $\phi^{-1}$ of the set $\{z=x$ : $-(n+1 / 3) h \leq x \leq(n+1 / 3) h\}$ with deleted segments of length $2 \varepsilon$ centered at points $k h, k=-n(1) n$, and $\phi(t)$. Multiplying both sides of (B.11) by $\phi^{\prime}(\tau) / \pi[\phi(\tau)-\phi(t)]$ and integrating over $L_{n}^{\varepsilon}$ with respect to $\tau$, we get

$$
\begin{aligned}
\frac{1}{\pi i} \int_{L_{n}^{\varepsilon}} & \frac{F(\tau) d \tau}{\tau-t}-\sum_{k=-n}^{n} \frac{F\left(z_{k}\right) h(-1)^{k}}{\phi^{\prime}\left(z_{k}\right) \pi i\left(t-z_{k}\right)} \\
& \times \int_{L_{n}^{\varepsilon}} \frac{1}{\pi}\left\{\frac{\sin [\pi \phi(\tau) / h] \phi^{\prime}(\tau)}{\phi(\tau)-\phi(t)}-\frac{\sin [\pi \phi(\tau) / h] \phi^{\prime}(\tau)}{\phi(\tau)-k h}\right\} d \tau \\
= & -\frac{1}{2 \pi}\left\{\frac{1}{\pi} \int_{L_{n}^{\varepsilon}} \frac{\sin [\pi \phi(\tau) / h] \phi^{\prime}(\tau) d \tau}{\phi(\tau)-\phi(t)} \int_{l_{n, Y}} \frac{F(z) d z}{(z-t) \sin [\pi \phi(z) / h]}\right. \\
& \left.+\int_{l_{n, Y}} \frac{1}{\pi} \int_{L_{n}^{\varepsilon}} \frac{\sin [\pi \phi(\tau) / h] \phi^{\prime}(\tau) d \tau}{\phi(z)-\phi(\tau)} \frac{F(z) d z}{(z-t) \sin [\pi \phi(z) / h]}\right\} .
\end{aligned}
$$

In the last integral in (B.12) we interchanged the order of integration, which is possible, since by (B.9), $|\phi(z)-\phi(\tau)| \geq \min (h / 3, Y)>0$ for all $z \in l_{n, Y}$ and all $\tau \in L_{n}$. Making a change of variables and letting $\varepsilon$ go to zero in (B.12), we obtain

$$
\frac{1}{\pi i} f_{L_{n}} \frac{F(\tau) d \tau}{\tau-t}-\sum_{k=-n}^{n} \frac{F\left(z_{k}\right)}{\phi^{\prime}\left(z_{k}\right)} \frac{h(-1)^{k}}{\pi i\left(t-z_{k}\right)} \frac{1}{\pi} f_{-N_{n}}^{N_{n}}\left\{\frac{\sin [\pi x / h]}{x-\phi(t)}-\frac{\sin [\pi x / h]}{x-k h}\right\} d x
$$

$$
\begin{aligned}
&=\frac{-1}{2 \pi}\left\{\frac{1}{\pi} \int_{-N_{n}}^{N_{n}}\right. \frac{\sin [\pi x / h] d x}{x-\phi(t)} \int_{l_{n, Y}} \frac{F(z) d z}{(z-t) \sin [\pi \phi(z) / h]} \\
&\left.\quad+\int_{l_{n, Y}} \frac{1}{\pi} \int_{-N_{n}}^{N_{n}} \frac{\sin [\pi x / h] d x}{\phi(z)-x} \frac{F(z) d z}{(z-t) \sin [\pi \phi(z) / h]}\right\},
\end{aligned}
$$

where $N_{n}=(n+1 / 3) h$.

Now we let $n$ approach $\infty$ and analyze (B.13). On the left-hand side we introduce

$$
\begin{aligned}
\delta_{n} & =\frac{1}{\pi}\left(f_{-\infty}^{\infty}-f_{-N_{n}}^{N_{n}}\right) \frac{\sin [\pi x / h] d x}{x-\phi(t)}, \\
\delta_{n, k} & =\frac{1}{\pi}\left(f_{-\infty}^{\infty}-f_{-N_{n}}^{N_{n}}\right) \frac{\sin [\pi x / h] d x}{x-k h},
\end{aligned}
$$

where in the second expression in (B.14), $k=-n(1) n$. Clearly,

$$
\lim _{n \rightarrow \infty} \delta_{n}=0 \text {, }
$$

but more importantly, it can be shown that

$$
\lim _{n \rightarrow \infty} \max _{-n / 2 \leq k \leq n / 2}\left|\delta_{n, k}\right|=0
$$


and also that there exists a constant $C_{1}>0$ such that

$$
\max _{-n \leq k \leq n}\left|\delta_{n, k}\right| \leq C_{1} \quad \text { as } n \rightarrow \infty .
$$

Using (B.14), the identity

$$
f_{-\infty}^{\infty} \frac{\sin [\pi x / h] d x}{x-x^{*}}=\pi \cos \left(\pi x^{*} / h\right) \quad \text { for } x^{*} \in R,
$$

and the definition of $t_{k}(z)$, the left-hand side of (B.13) can be rewritten as

$$
\frac{1}{\pi i} f_{L_{n}} \frac{F(\tau) d \tau}{\tau-t}-\sum_{k=-n}^{n} F\left(z_{k}\right) t_{k}(t)+\sum_{k=-n}^{n} \frac{F\left(z_{k}\right)}{\phi^{\prime}\left(z_{k}\right)} \frac{h(-1)^{k}}{\pi i\left(t-z_{k}\right)}\left(\delta_{n}-\delta_{n, k}\right) .
$$

It follows now from (B.15)-(B.17) and (5.5) that as $n \rightarrow \infty$ the expression (B.19), and hence the left-hand side of (B.13), becomes

$$
S F(t)-\sum_{k \in \mathbf{Z}} F\left(z_{k}\right) t_{k}(t)
$$

Now we turn to the right-hand side of (B.13). Since $|\sin [\pi \phi(z) / h]| \geq \sqrt{3} / 2$ for all $z \in l_{n, Y}^{2}$, it follows from (B.18), the definition of $l_{n, Y}$ in (B.9), (4.3), (4.5) and (4.6) that as $n \rightarrow \infty$ the first term on the right-hand side of (B.13) approaches

$$
-\frac{1}{2 \pi} \cos [\pi \phi(t) / h] \int_{\phi^{-1}\left(L_{Y}\right)} \frac{F(z) d z}{(z-t) \sin [\pi \phi(z) / h]} .
$$

Thus, it only remains to consider the second term on the right-hand side of (B.13). It can be shown that there exists a constant $C_{2}>0$ such that for all $z \in l_{n, Y}^{2}$,

$$
\left|\int_{-N_{n}}^{N_{n}} \frac{\sin [\pi x / h] d x}{\phi(z)-x}\right| \leq C_{2}
$$

uniformly as $n \rightarrow \infty$. Hence it follows by the same argument as the one used above that the part of the integral corresponding to $l_{n, Y}^{2}$ vanishes as $n \rightarrow \infty$. Let us rewrite the contribution from $l_{n, Y}^{1}$ as follows:

$$
-\frac{1}{2 \pi} \int_{\phi^{-1}\left(L_{Y}\right)} \chi_{n, Y} \frac{1}{\pi} \int_{-N_{n}}^{N_{n}} \frac{\sin [\pi x / h] d x}{\phi(z)-x} \frac{F(z) d z}{(z-t) \sin [\pi \phi(z) / h]},
$$

where $\chi_{n, Y}$ in (B.23) is the characteristic function of $l_{n, Y}^{1}$. Similarly to the case of (B.22), it can now be shown that there exists a constant $C_{3}>0$ such that for all $z \in \phi^{-1}\left(L_{Y}\right)$,

$$
\left|\int_{-N_{n}}^{N_{n}} \frac{\sin [\pi x / h] d x}{\phi(z)-x}\right| \leq C_{3}
$$

uniformly as $n \rightarrow \infty$. Therefore, from (B.24), Lebesgue's dominated convergence theorem and the identity

$$
\int_{-\infty}^{\infty} \frac{\sin [\pi x / h] d x}{x-z}=\pi \exp [(i \pi z / h) \operatorname{sgn} \operatorname{Im} z], \quad z \in \mathbf{C} \backslash \mathbf{R},
$$

it follows that as $n \rightarrow \infty$ the expression (B.23) approaches

$$
\frac{1}{2 \pi} \int_{\phi^{-1}\left(L_{Y}\right)} \frac{\exp [(i \pi \phi(z) / h) \operatorname{sgn} \operatorname{Im} \phi(z)] F(z) d z}{(z-t) \sin [\pi \phi(z) / h]} .
$$


Finally, combining all results from (B.20), (B.21) and (B.26), we obtain

$$
\begin{aligned}
S F(t) & -\sum_{n \in \mathbf{Z}} F\left(z_{n}\right) t_{n}(t) \\
& =\frac{1}{2 \pi} \int_{\phi^{-1}\left(L_{Y}\right)} \frac{F(z)\{\exp [(i \pi \phi(z) / h) \operatorname{sgn} \operatorname{Im} \phi(z)]-\cos [\pi \phi(t) / h]\} d z}{(z-t) \sin [\pi \phi(z) / h]} .
\end{aligned}
$$

The error bound (5.8) now follows from (B.27) by bounding the contour integral, taking the $\liminf \operatorname{in}_{Y \rightarrow d^{-}}$and making use of the inequality

$$
|\sin [\pi \phi(z) / h]| \geq \sinh |\pi Y / h|, \quad z \in \phi^{-1}\left(L_{Y}\right) .
$$

The proof of Theorem 5.2 for the case when (5.6) is satisfied instead of (5.5) is similar, and we will not give it in detail. We only note in this case that by the first inequality in (B.5) and the estimate (5.4), the expression in (B.19), i.e., the left-hand side of (B.13), approaches the limiting form (B.20) as $n \rightarrow \infty$. This again yields (B.27), from which we obtain the estimate (5.8) as above.

Department of Mathematics

University of Utah

Salt Lake City, Utah 84112

1. P. M. Anselone, Collectively Compact Operator Approximation Theory and Applications to Integral Equations, Prentice-Hall, Englewood Cliffs, N.J., 1971.

2. K. E. ATKINSON, A Survey of Numerical Methods for the Solution of Fredholm Integral Equations of the Second Kind, SIAM, Philadelphia, Pa., 1976.

3. M. L. DOW \& D. ElliotT, "The numerical solution of singular integral equations over $(-1,1)$," SIAM J. Numer. Anal., v. 16, 1979, pp. 115-134.

4. D. Elliot T, "The classical collocation method for singular integral equations," SIAM J. Numer. Anal., v. 19, 1982, pp. 816-832.

5. D. ElliotT, "Rates of convergence for the method of classical collocation for solving singular integral equations," SIAM J. Numer. Anal., v. 21, 1984, pp. 136-148.

6. D. Elliott \& F. STENGER, Sinc Method of Solution of Singular Integral Equations, IMACS Symposium on Numerical Solution of Singular Integral Equations, IMACS, 1984, pp. 27-35.

7. G. M. Goluzin, Geometric Theory of Functions of a Complex Variable, Transl. Math. Monos., vol. 26, Amer. Math. Soc., Providence, R.I., 1969.

8. N. I. IOAKIMIDIS, "On the weighted Galerkin method of numerical solution of Cauchy type singular integral equations," SIAM J. Numer. Anal., v. 18, 1981, pp. 1120-1127.

9. N. I. IOAKIMIDIS, "Further convergence results for the weighted Galerkin method of numerical solution of Cauchy-type singular integral equations," Math. Comp., v. 41, 1983, pp. 79-85.

10. P. LiNZ, "An analysis of a method for solving singular integral equations," $B I T$, v. 17, 1977, pp. 329-337.

11. G. MIEL, "On the Galerkin and collocation methods for a Cauchy singular integral equation," SIAM J. Numer. Anal., v. 23, 1986, pp. 135 143.

12. N. I. MUSKhelishVili, Singular Integral Equations, Noordhoff, Groningen, 1958.

13. F. STENGER, "Numerical methods based on Whittaker cardinal, or Sinc functions," SIAM Rev., v. 23, 1981, pp. $165-224$.

14. K. S. ThомAS, "Galerkin methods for singular integral equations," Math. Comp., v. 36, 1981, pp. 193205.

15. J. H. Wilkinson, Rounding Errors in Algebraic Processes, Her Majesty's Stationery Office, London, 1963. 